JOURNAL OF THE AMERICAN MATHEMATICAL SOCIETY

Volume 10, Number 4, October 1997, Pages 761-796

S 0894-0347(97)00244-0

\title{
HARMONIC FUNCTIONS OF MAXIMAL GROWTH: INVERTIBILITY AND CYCLICITY IN BERGMAN SPACES
}

\author{
ALEXANDER BORICHEV AND HÅKAN HEDENMALM
}

\section{INTRODUCTION AND MAIN RESULTS}

In the Gelfand theory of commutative Banach algebras with unit, an element generates a dense ideal if and only if it is invertible, in which case its Gelfand transform has no zeros, and the ideal it generates is the whole algebra. With varying degrees of success, efforts have been made to extend the validity of this result to the more general context of Banach (or even topological vector) spaces of functions. To do this properly, we need the space $M(X)$ of multipliers on the given space $X$ : a function $f$, defined on the same underlying region, is a multiplier on $X$ if $f X$ is contained in $X$. If $X$ contains the constant functions, then $M(X) \subset X$. In the Banach algebra case, $M(X)=X$. Observe that $M(X)$ forms an algebra, and if $X$ is a Banach space, then $M(X)$ is a Banach algebra. An element $f \in X$ is said to be multiplier-cyclic in $X$ if $f M(X)$ is dense in $X$. In the Banach algebra case, an element is multiplier-cyclic if and only if it is invertible, or, which is the same, its Gelfand transform lacks zeros.

We illustrate the situation with two well-known spaces: $L^{2}$, the square integrable functions on the unit circle $\mathbb{T}$, and $H^{2}$, the Hardy space on the unit disk $\mathbb{D}$. For $X=L^{2}$, the multiplier space is $M\left(L^{2}\right)=L^{\infty}$, the space of essentially bounded functions on $\mathbb{T}$. A function in $L^{2}$ is multiplier-cyclic in $L^{2}$ if and only if its zero set is a null set with respect to arc length measure on $\mathbb{T}$. For $X=H^{2}$, the multiplier space is $M\left(H^{2}\right)=H^{\infty}$, the bounded analytic functions on $\mathbb{D}$. By Beurling's invariant subspace theorem, a function is multiplier-cyclic if and only if it is outer. Invertible functions in $H^{2}$ are all outer, but not all outer functions are invertible.

The above-mentioned results suggest that invertible elements are multipliercyclic in a more general setting. However, this intuition is wrong: as we leave the Nevanlinna space setting, new phenomena appear, which may prevent invertible elements from being multiplier-cyclic. What makes this possible is a curious combination of growth and decrease. We shall supply examples of functions in the Bergman spaces (and in certain uniform Bergman spaces) on the unit disk which are

Received by the editors July 18, 1996 and, in revised form, March 25, 1997.

1991 Mathematics Subject Classification. Primary 30H05, $46 \mathrm{E} 15$.

Key words and phrases. Bergman spaces, cyclic vectors, canonical divisors.

The research of both authors was supported in part by the Swedish Natural Science Research Council. The second author was also supported by the 1992 Wallenberg Prize from the Swedish Mathematical Society.

The paper was written when the authors worked at the Uppsala University, Sweden, and visited MSRI, USA. 
invertible, but not multiplier-cyclic (neither are they polynomial-cyclic; see the definition below). This answers in the negative questions raised by Shapiro, Nikolskilu, Shields, Korenblum, Brown, and Frankfurt.

We also need the concept of polynomial-cyclicity. Let $X$ be a complex-linear topological space of analytic functions on $\mathbb{D}$. Suppose $X$ is closed under multiplication by the coordinate function $z$, so that the multiplier space $M(X)$ contains the polynomials. We say that an element $f \in X$ is polynomial-cyclic (the term weakly invertible is also common) in $X$ if the set of polynomial multiples of $f$ is dense in $X$. Generally speaking, it is harder for a function to be polynomial-cyclic than multiplier-cyclic, although in many cases the two concepts coincide, as in the case of $H^{2}$ above.

Let $d S(z)=\pi^{-1} d x d y(z=x+i y)$ be normalized Lebesgue area measure, and $p$ a real parameter, $0<p<+\infty$. In this paper we look at the Bergman space $L_{a}^{p}$, which consists of all functions $f$ holomorphic on $\mathbb{D}$, subject to the norm boundedness condition

$$
\|f\|_{L_{a}^{p}}=\left(\int_{\mathbb{D}}|f(z)|^{p} d S(z)\right)^{1 / p}<+\infty .
$$

It is a Hilbert space for $p=2$, a Banach space for $1 \leq p<+\infty$, and a complete metric space for $0<p<1$ (the invariant metric being given by the $p$-th power of the norm expression). We shall also be interested in the related spaces $A^{-p}, A_{0}^{-p}$, and $A^{-\infty}$, defined as follows. The space $A^{-p}$ is the (non-separable) Banach space of all functions $f$ holomorphic on $\mathbb{D}$ that satisfy

$$
\|f\|_{A^{-p}}=\sup \left\{(1-|z|)^{p}|f(z)|: z \in \mathbb{D}\right\}<+\infty,
$$

and $A_{0}^{-p}$ is the closure of the polynomials in $A^{-p}$. The space $A_{0}^{-p}$ is supplied with the norm topology of $A^{-p}$, which makes it a Banach space, too. One shows that a function $f \in A^{-p}$ is in $A_{0}^{-p}$ if and only if

$$
(1-|z|)^{p}|f(z)| \rightarrow 0 \quad \text { as } \quad|z| \rightarrow 1 .
$$

As the parameter $p$ increases, the spaces $A^{-p}$ and $A_{0}^{-p}$ get larger, and $L_{a}^{p}$ gets smaller. We will refer to the $A^{-p}$ and $A_{0}^{-p}$ as uniform Bergman spaces. The Korenblum space $A^{-\infty}$ is the union of all $A^{-p}$, with the inductive limit topology. It is also the union of all Bergman spaces $L_{a}^{p}$, as well as the union of all the $A_{0}^{-p}$, and the various inductive limit topologies it inherits this way are the same.

The space of multipliers on any one of the above Bergman or uniform Bergman spaces is $H^{\infty}$. The Korenblum space $A^{-\infty}$ is a topological algebra, and as such its multiplier space is $A^{-\infty}$ itself.

No single function in the space $A^{-p}$ is polynomial-cyclic, because $A^{-p}$ is nonseparable. A slightly more sophisticated argument is required to show that there are no multiplier-cyclic functions either. Let $f \in A^{-p}$; one readily checks that there exists a sequence $\left\{z_{j}\right\}_{j}$ of points in $\mathbb{D}$ tending to the unit circle such that $(1-|z|)^{p}|f(z)|$ approaches 0 along it. Any function in the norm closure in $A^{-p}$ of $f H^{\infty}$ must also have this property for the same sequence. However, there are functions $g \in A^{-p}$ that do not, so $f$ cannot be multiplier-cyclic. The existence of such functions $g$ follows from the following argument: by thinning out the sequence $\left\{z_{j}\right\}_{j}$, what remains can be made interpolating for $A^{-p}$, so that there are functions growing maximally there (see, for instance, [18]). This suggests the concept of weak-star cyclicity: a function in $A^{-p}$ is said to be weak-star multiplier-cyclic if 
$f H^{\infty}$ is weak-star dense in $A^{-p}$, and weak-star polynomial-cyclic if the polynomial multiples of $f$ are weak-star dense in $A^{-p}$. The weak-star topology refers to a fixed predual, to be supplied shortly.

Let $L^{\infty,-p}$ be the space of (equivalence classes of) complex-valued Lebesgue measurable functions $f$ on $\mathbb{D}$ with $(1-|z|)^{p} f(z)$ in $L^{\infty}(\mathbb{D})$, so that $A^{-p}$ is the intersection of $L^{\infty,-p}$ with the holomorphic functions on $\mathbb{D}$. The predual of $L^{\infty,-p}$ is the space $L^{1, p}$ of all complex-valued functions $g$ with $(1-|z|)^{-p} g(z)$ in $L^{1}(\mathbb{D})$. It gives $L^{\infty,-p}$ a weak-star topology. The space $A^{-p}$ is a weak-star closed subspace of $L^{\infty,-p}$, and as such it has as predual the quotient space $L^{1, p} /^{\perp} A^{-p}$, where ${ }^{\perp} A^{-p}$ is the preannihilator of $A^{-p}$. One shows that a sequence $\left\{f_{k}\right\}_{k}$ of functions in $A^{-p}$ converges weak-star to $f \in A^{-p}$ if and only if $f_{k}(z) \rightarrow f(z)$ uniformly on compact subsets of $\mathbb{D}$ as $k \rightarrow+\infty$, and $\sup _{k}\left\|f_{k}\right\|_{A^{-p}}<+\infty$. A set $Y \subset A^{-p}$ is said to be weak-star sequentially closed if all weak-star convergent sequences have limit points in $Y$. Standard functional analysis machinery (the Banach-Alaoglu theorem, Banach's metrizability theorem for the weak-star topology on the closed unit ball, and the Krein-Shmulian theorem; see [6]) together with the fact that $L^{1, p}$ is separable, shows that a convex subset $Y$ of $A^{-p}$ is weak-star closed if and only if it is weak-star sequentially closed.

A word should now be said about the relationship between the concepts of multiplier-cyclicity and polynomial-cyclicity in the context of our Bergman, uniform Bergman, and Korenblum spaces. By approximating functions in $H^{\infty}$ by polynomials, one can show that in the spaces $L_{a}^{p}$ and $A_{0}^{-p}$, a function is polynomialcyclic if and only if it is multiplier-cyclic. Moreover, the same type of argument shows that in the space $A^{-p}$, a function is weak-star polynomial-cyclic if and only if it is weak-star multiplier-cyclic. Functions in $A^{-\infty}$ may be approximated by polynomials, so that multiplier-cyclicity is the same as polynomial-cyclicity for the topological algebra $A^{-\infty}$ as well. Since these concepts coincide in all cases we are concerned with, we shall drop the distinction between them, and talk only about cyclicity and cyclic functions (and add the prefix weak-star when needed).

If, as is the case for the above-defined Bergman, uniform Bergman, and Korenblum spaces, point evaluation functionals at points of $\mathbb{D}$ are continuous and non-trivial, then an obvious necessary condition for a function to be cyclic is that it should vanish nowhere on $\mathbb{D}$. For the space $A^{-\infty}$, Boris Korenblum's factorization theory [13], [14] offers a complete description of the cyclic vectors based on the notion of $\varkappa$-singular measure.

Theorem A. An element $f$ in $A^{-\infty}$ is cyclic if and only if it vanishes nowhere on $\mathbb{D}$, and the $\varkappa$-singular measure associated with $f$ equals 0 .

Clearly, invertible functions in $A^{-\infty}$ are cyclic in $A^{-\infty}$. Note that $f \in A^{-\infty}$ is invertible in $A^{-\infty}$ if and only if for some positive constants $C, N$,

$$
|f(z)| \geq C(1-|z|)^{N}, \quad z \in \mathbb{D} .
$$

Concerning cyclicity in $A_{0}^{-p}$, the following assertion is shown in [4].

Theorem B. $(0<p<+\infty)$ If $f \in A_{0}^{-p}$ and $f$ is cyclic in $A^{-\infty}$, then $f$ is cyclic in every $A_{0}^{-q}$, with $p<q<+\infty$.

It is clear that cyclicity in $A_{0}^{-p}$ implies cyclicity in the larger spaces $A_{0}^{-q}$, for $p<q<+\infty$. Leon Brown and Korenblum [4] raised the question as to whether 
the two conditions that $f \in A_{0}^{-p}$ and $f$ is cyclic (or invertible) in $A^{-\infty}$ imply that $f$ is cyclic in $A_{0}^{-p}$. Here this question is answered in the negative.

Theorem 1.1. $(0<p, q<+\infty)$ There exists a function $f$ in $A_{0}^{-p}$ such that $1 / f \in$ $A^{-q}$, but $f$ is not cyclic in $A_{0}^{-p}$.

The first example of a Banach space of analytic functions in $\mathbb{D}$ where invertibility does not imply cyclicity was constructed by Faizo Shamoyan [19] (see also [23], Commentary).

The weak-star topology of $A^{-p}$ is non-metrizable, so the set of weak-star sequential limits of elements of a given subset need not be weak-star closed. To get the weak-star closure, the operation of taking sequential limits may need to be applied many times. The following example illustrates this point.

Theorem 1.2. $(0<p<+\infty)$ There exists an outer function $f$ in the Nevanlinna class, such that $f \in A^{-p}$ and $1 / f \in \bigcap_{q} A^{-q}$, where the intersection is over all $q$ with $0<q<+\infty$, but the function 1 is not a weak-star sequential limit of polynomial multiples of $f$.

In the previous theorem, the constant function 1 does belong to the weak-star closure of the set of polynomial multiples of $f$, as $f$ can be shown to be weak-star cyclic in $A^{-p}$. Nevertheless, a genuine analog of Theorem 1.1 can be obtained.

Theorem 1.3. $(0<p, q<+\infty)$ There exists a function $f$ in $A^{-p}$ such that $1 / f \in$ $A^{-q}$, but $f$ fails to be weak-star cyclic in $A^{-p}$.

The first result analogous to Theorem B for the Bergman space $L_{a}^{p}$ was obtained by Harold Shapiro [20], [21] (Shapiro's original result was for $q=2$, but according to [4], it holds generally).

Theorem C. $(0<p<+\infty)$ If $f \in L_{a}^{p}$ and $1 / f \in A^{-\infty}$, then $f$ is cyclic in every larger $L_{a}^{q}, 0<q<p$.

By [4], the following analog of Theorem B holds for the spaces $L_{a}^{p}$.

Theorem D. $(0<p<+\infty)$ If $f \in L_{a}^{p}$ and $f$ is cyclic in $A^{-\infty}$, then $f$ is cyclic in every larger $L_{a}^{q}, 0<q<p$.

The question of whether the conditions

(a) $f \in L_{a}^{p}, 1 / f \in A^{-\infty}$ or

(b) $f \in L_{a}^{p}, f$ is cyclic in $A^{-\infty}$

imply that $f$ is cyclic in $L_{a}^{p}$ was first raised in [20], and later in [17], p. 93, [1], [22], Question 25, [15], [23], [24], Question 5, and [12]. A variant of this problem is whether the conditions $f \in L_{a}^{p}, 1 / f \in L_{a}^{q}$ imply that $f$ is cyclic in $L_{a}^{p}$. When $q=p$, the question is whether invertibility in $L_{a}^{p}$ implies cyclicity (weak invertibility) in $L_{a}^{p}$. In this form, it was mentioned in [17], p. 93, [22], Question 25', [23], and [9].

We construct an example answering these questions in the negative.

Theorem 1.4. $(0<p, q<+\infty)$ There exists a function $f$ in $L_{a}^{p}$ which is not cyclic in $L_{a}^{p}$, although $1 / f \in A^{-q}$.

In [16], Korenblum introduced the notion of an $L_{a}^{p}$-outer function, and proved that a cyclic function necessarily is $L_{a}^{p}$-outer. Recent work by Alexandru Aleman, Stefan Richter, and Carl Sundberg [2] has shown that the $L_{a}^{p}$-outer functions are 
precisely the cyclic functions. Thus Theorem 1.4 answers Question 2 in [16] in the negative: there are functions in $L_{a}^{p}$ that are cyclic in $A^{-\infty}$, and yet not $L_{a}^{p}$-outer.

The underlying idea behind the constructions of non-cyclic functions in the function spaces considered here is that not only decrease, but also growth can be an impediment to cyclicity. The functions constructed will be in some sense extremal in the given space. To be more precise, the set of points $E$ in the unit disk where the function is "maximally" big should be pretty massive: in Corollary 5.1, the closure of $E$ contains the unit circle $\mathbb{T}$, in Corollary 7.1, $E$ is dominating for $H^{\infty}$, and in Corollaries 9.1 and $11.1, \mathbb{T}$ has zero harmonic measure in $\mathbb{D} \backslash E$. We now suggest a heuristic argument explaining why the indicated growth makes the given function $f$ non-cyclic. Let $\left\{h_{n}\right\}_{n}$ be a sequence of functions in $H^{\infty}$, such that $f h_{n}$ converges to some element $g$ in the given space $X$. The fact that $f$ is "maximally big" on $E$ makes $h_{n}$ grow modestly on $E$, and since $E$ is massive, $h_{n}$ must grow modestly throughout $\mathbb{D}$, with an estimate that is uniform in $n$. Given that $f(z)$ tends to 0 at a certain speed along a sequence tending to $\mathbb{T}$, a little faster than the uniform bound in $n$ of $h_{n}(z)$ tends to infinity, we get that the limit function $g$ tends to 0 along the sequence. Then $f$ is not cyclic, because we cannot get the constant function 1 in the closure of $f H^{\infty}$.

All the proofs of the theorems (1.1-1.4) stated here involve highly lacunary constructions of harmonic functions. The one associated with Theorem 1.2 resembles an example by Nikolaŭ Nikolskiü [17], p. 84, Theorem 2. Like us, Nikolskiı̆ constructs a certain harmonic function as a Poisson integral of a norm convergent sum of Borel measures on $\mathbb{T}$. Our other theorems $(1.1,1.3,1.4)$ are based on the same idea, only there, the sum of the Borel measures is norm divergent, though convergent in the space of distributions.

The analytic functions that we construct exhibit bad boundary behavior everywhere on the unit circle. However, the constructions do modify so as to supply non-cyclic functions that extend analytically across a given arc of $\mathbb{T}$ (if the arc has length strictly less than that of the circle).

In the Hardy spaces $H^{p}$, a function is non-cyclic if and only if it has a nontrivial inner factor, and the latter implies that either the function has zeros, or that it has a non-trivial singular inner factor. That the function has a non-trivial singular inner factor means that it decreases at a critical rate near a subset of the unit circle with zero length. Here we have found that a function in the Bergman or uniform Bergman spaces can be non-cyclic due to growth. Growth and critical decrease near a small set may combine forces, as we shall see. Let $g$ be a function in a Bergman or uniform Bergman space, and let $u$ be a singular inner function. It is natural to ask for which $u$ the function $u g$ generates the same invariant subspace as $g$ does. If $g$ is the constant function 1, or, more generally, if $g$ belongs to a slightly smaller space (as in Theorems B and D), and is cyclic in $A^{-\infty}$, then the answer is that $u g$ generates the same invariant subspace as $g$ does (that is, the whole space) if and only if the singular measure associated with $u$ places no mass on any Beurling-Carleson set of zero length. However, if $g$ grows too fast to belong to a slightly smaller space, the behavior of the singular measure associated with $u$ on sets larger than Beurling-Carleson ones may become essential. For instance, let us look at the space $A^{-p}$, and let $g$ be the extremally growing function which is constructed for Theorem 1.3, and has the property that it generates the weak-star closed invariant subspace $g H^{\infty}$ in $A^{-p}$. Then $u g$ generates the weak-star closed invariant subspace $u g H^{\infty}$, which coincides with $g H^{\infty}$ if and only if $u$ is constant 
(which can be normalized so that $u=1$ ). That is, the singular measure associated with $u$ must place zero mass on every Borel measurable subset of zero length. We hope that this example will inspire further work toward a complete description of the cyclic functions.

The exposition of the paper is as follows. Theorems 1.1-1.4 are proved in Section 12. The proofs rely on explicit constructions of harmonic functions, which are carried out in Sections 4, 6, 8, and 10. Each construction is labeled I, II, III, and IV; I relates to Theorem 1.2, II to Theorem 1.3, III to Theorem 1.1, and IV to Theorem 1.4. The harmonic function constructions lead to the existence of certain zero-free holomorphic functions by taking exponentials of the harmonic functions plus $i$ times their harmonic conjugates, and the properties of them that we need are stated in Sections 5, 7, 9, and 11. In Section 13, we apply the results to the recent factorization theory in the Bergman spaces based on extremal functions (inner divisors) [11], [7], [8], [12], [2]. Technical results on harmonic functions needed in the constructions are supplied in Sections 2 and 3.

The results contained in this paper were announced earlier in [3].

\section{Preliminaries on harmonic FunCtions}

For $1 \leq p<+\infty$, let $h^{p}(\mathbb{D})$ denote the Banach space of complex-valued functions $f$ harmonic in the disk $\mathbb{D}$, with

$$
\|f\|_{h^{p}}=\sup _{0<r<1}\left(\frac{1}{2 \pi} \int_{-\pi}^{\pi}\left|f\left(r e^{i \theta}\right)\right|^{p} d \theta\right)^{1 / p}<+\infty .
$$

Also, let $h^{\infty}(\mathbb{D})$ be the Banach space of complex-valued bounded harmonic functions on $\mathbb{D}$, with norm

$$
\|f\|_{h^{\infty}}=\sup \{|f(z)|: z \in \mathbb{D}\}<+\infty .
$$

For a finite Borel measure $\mu$ on $\mathbb{T}$, its Poisson integral is the function

$$
P \mu(z)=\frac{1}{2 \pi} \int_{\mathbb{T}} P(z, \zeta) d \mu(\zeta), \quad z \in \mathbb{D},
$$

where

$$
P(z, \zeta)=\frac{1-|z|^{2}}{|\zeta-z|^{2}}, \quad(z, \zeta) \in \mathbb{D} \times \mathbb{T},
$$

is the Poisson kernel. For functions $f \in L^{1}(\mathbb{T})$, we write $P f$ instead of $P \mu_{f}$, with $d \mu_{f}\left(e^{i \theta}\right)=f\left(e^{i \theta}\right) d \theta$. The function $P f$ extends $f$ harmonically to the interior $\mathbb{D}$. It is well known that a harmonic function belongs to $h^{1}(\mathbb{D})$ if and only if it is the Poisson integral of a finite Borel measure. Moreover, for $1<p \leq+\infty$, the space $h^{p}(\mathbb{D})$ coincides with the space of Poisson integrals of $L^{p}(\mathbb{T})$ functions. We shall need to estimate the size of the Poisson integral of a measure with small support. For a Borel measure $\mu$ on $\mathbb{T},\|\mu\|$ stands for its total variation.

Lemma 2.1. For $0 \leq t \leq 2 \pi$, let $J(t)$ be the closed arc connecting the point 1 with $e^{i t}$, running counter-clockwise. If $\mu$ is a complex-valued Borel measure supported on $J(\beta)$, for some $\beta, 0<\beta<2 \pi$, which has $\|\mu\|=1$ and $\mu(\mathbb{T})=0$, then

$$
|P \mu(z)| \leq \frac{\beta}{2 \pi} \frac{1-|z|^{2}}{d(z, J(\beta))^{3}}, \quad z \in \mathbb{D},
$$


where $d$ is the Euclidean metric. In particular,

$$
|P \mu(z)| \leq \frac{1}{\pi} \frac{\beta}{(1-|z|)^{2}}, \quad z \in \mathbb{D} .
$$

Proof. Let $M\left(e^{i \theta}\right)$ be the function $\mu(J(\theta))$, which is well defined at 1 because $\mu(\mathbb{T})=0$. The function $M$ is supported on $J(\beta)$, and its supremum norm has the bound $\|M\|_{L^{\infty}} \leq 1 / 2$. Integration by parts gives

$$
\int_{-\pi}^{\pi} P\left(z, e^{i \theta}\right) d \mu\left(e^{i \theta}\right)=\left(1-|z|^{2}\right) \int_{-\pi}^{\pi} M\left(e^{i \theta}\right) \frac{d}{d \theta}\left|e^{i \theta}-z\right|^{-2} d \theta,
$$

and since

$$
\left|\frac{d}{d \theta}\right| e^{i \theta}-\left.z\right|^{-2} \mid \leq \frac{2}{\left|e^{i \theta}-z\right|^{3}}, \quad z \in \mathbb{D}
$$

we get

$$
\frac{1}{2 \pi}\left|\int_{-\pi}^{\pi} P\left(z, e^{i \theta}\right) d \mu\left(e^{i \theta}\right)\right| \leq \frac{1-|z|^{2}}{\pi} \int_{0}^{\beta} \frac{\|M\|_{L^{\infty}}}{\left|e^{i \theta}-z\right|^{3}} d \theta \leq \frac{\beta}{2 \pi} \frac{1-|z|^{2}}{d(z, J(\beta))^{3}}, \quad z \in \mathbb{D} .
$$

The proof is complete.

\section{BUILDING BLOCKS}

Our basic building blocks in later constructions will be the functions $\Phi_{\alpha, \beta}$, for $0<\alpha<\beta \leq 2 \pi$, which are defined as

$$
\Phi_{\alpha, \beta}(z)=\frac{1}{\alpha} \omega(z, I(\alpha), \mathbb{D})-\frac{1}{\beta} \omega(z, I(\beta), \mathbb{D}), \quad z \in \mathbb{D},
$$

where $\omega$ is harmonic measure, and for $0<\tau \leq 2 \pi, I(\tau)$ is the arc

$$
I(\tau)=\left\{e^{i \theta}: \theta \in\left[-\frac{1}{2} \tau, \frac{1}{2} \tau\right]\right\} .
$$

In case $\beta=2 \pi$, we write $\Phi_{\alpha}$ in place of $\Phi_{\alpha, 2 \pi}$ :

$$
\Phi_{\alpha}(z)=\frac{1}{\alpha} \omega(z, I(\alpha), \mathbb{D})-\frac{1}{2 \pi}, \quad z \in \mathbb{D} .
$$

We extend the function $\Phi_{\alpha, \beta}$ to the boundary $\mathbb{T}$ by declaring

$$
\Phi_{\alpha, \beta}(z)=\left\{\begin{array}{l}
0, \quad z \in \mathbb{T} \backslash I(\beta), \\
-1 / \beta, \quad z \in I(\beta) \backslash I(\alpha), \\
1 / \alpha-1 / \beta, \quad z \in I(\alpha) .
\end{array}\right.
$$

One checks that $\Phi_{\alpha, \beta}(0)=0$. According to [10], pp. 41-42,

$$
\omega(z, I(\alpha), \mathbb{D})=\frac{1}{\pi} \arg \left(\frac{e^{i \alpha / 2}-z}{e^{-i \alpha / 2}-z}\right)-\frac{\alpha}{2 \pi}, \quad z \in \mathbb{D},
$$

with a suitable choice of the argument function, so that

$$
\Phi_{\alpha}(z)=\frac{1}{\pi \alpha} \arg \left(\frac{1-z e^{-i \alpha / 2}}{1-z e^{i \alpha / 2}}\right), \quad z \in \mathbb{D},
$$

and $\Phi_{\alpha, \beta}=\Phi_{\alpha}-\Phi_{\beta}$. For $0 \leq r<1$, put

$$
Q_{\alpha, \beta}(r)=\max \left\{\Phi_{\alpha, \beta}(z):|z|=r\right\},
$$

and extend the function continuously to $[0,1]$ by declaring $Q_{\alpha, \beta}(1)=1 / \alpha-1 / \beta$. Note that it is increasing in $r$, and has the property that the function $Q_{\alpha, \beta}\left(e^{t}\right)$ 
is convex on $]-\infty, 0]$. For geometric reasons, the above maximum is attained at $z=r$, and an explicit computation yields

$$
Q_{\alpha, \beta}(r)=\Phi_{\alpha, \beta}(r)=\frac{2}{\pi \alpha} \arctan \frac{r \sin \frac{1}{2} \alpha}{1-r \cos \frac{1}{2} \alpha}-\frac{2}{\pi \beta} \arctan \frac{r \sin \frac{1}{2} \beta}{1-r \cos \frac{1}{2} \beta} .
$$

For $0<\alpha \leq \pi$, the value at $r=\cos \frac{1}{2} \alpha$ is readily estimated, and the resulting estimate leads to

$$
\frac{1}{\alpha}-\frac{1}{\beta}-\frac{1}{2 \pi} \leq Q_{\alpha, \beta}\left(\cos \frac{1}{2} \alpha\right) \leq Q_{\alpha, \beta}(r)<\frac{1}{\alpha}-\frac{1}{\beta}, \quad \cos \frac{1}{2} \alpha \leq r<1 .
$$

To get a reasonable estimate on a longer interval, we proceed as follows. For $0<\alpha<\frac{1}{3} \pi$, we have $\cos \frac{1}{2} \alpha>1-\frac{1}{3} \sin \frac{1}{2} \alpha$, so that

$$
1<\frac{r \sin \frac{1}{2} \alpha}{1-r \cos \frac{1}{2} \alpha}, \quad 1-\frac{1}{2} \sin \frac{1}{2} \alpha \leq r<1,
$$

and consequently,

$$
\frac{1}{2 \alpha}-\frac{1}{\beta}<Q_{\alpha, \beta}(r)<\frac{1}{\alpha}-\frac{1}{\beta}, \quad 1-\frac{1}{2} \sin \frac{1}{2} \alpha \leq r<1 .
$$

The second derivative of $Q_{\alpha, \beta}(r)$ with respect to $r$ is

$$
Q_{\alpha, \beta}^{\prime \prime}(r)=\frac{4}{\pi \alpha} \frac{\left(\cos \frac{1}{2} \alpha-r\right) \sin \frac{1}{2} \alpha}{\left(1+r^{2}-2 r \cos \frac{1}{2} \alpha\right)^{2}}-\frac{4}{\pi \beta} \frac{\left(\cos \frac{1}{2} \beta-r\right) \sin \frac{1}{2} \beta}{\left(1+r^{2}-2 r \cos \frac{1}{2} \beta\right)^{2}} .
$$

One checks that for $0<\alpha<\beta<\pi, Q_{\alpha, \beta}^{\prime \prime}(r)>0$ on the interval $0<r<\cos \frac{1}{2} \alpha$, making $Q_{\alpha, \beta}$ convex there. In the special case $\beta=2 \pi$, we write $Q_{\alpha}$ in place of $Q_{\alpha, 2 \pi}$,

$$
Q_{\alpha}(r)=\frac{2}{\pi \alpha} \arctan \frac{r \sin \frac{1}{2} \alpha}{1-r \cos \frac{1}{2} \alpha},
$$

and observe that the above formula for the second derivative reduces to

$$
Q_{\alpha}^{\prime \prime}(r)=\frac{4}{\pi \alpha} \frac{\left(\cos \frac{1}{2} \alpha-r\right) \sin \frac{1}{2} \alpha}{\left(1+r^{2}-2 r \cos \frac{1}{2} \alpha\right)^{2}} .
$$

It follows that $Q_{\alpha}$ is convex on $\left[0, \cos \frac{1}{2} \alpha\right]$, and concave on $\left[\cos \frac{1}{2} \alpha, 1\right]$. The value at the inflexion point is

$$
Q_{\alpha}\left(\cos \frac{1}{2} \alpha\right)=\frac{2}{\pi \alpha} \arctan \frac{\sin \frac{1}{2} \alpha \cos \frac{1}{2} \alpha}{1-\cos ^{2} \frac{1}{2} \alpha}=\frac{1}{\alpha}-\frac{1}{\pi},
$$

so that by convexity $(0<\alpha<\pi)$,

$$
Q_{\alpha}(r) \leq \frac{\pi-\alpha}{\pi \alpha \cos \frac{1}{2} \alpha} r, \quad 0 \leq r \leq \cos \frac{1}{2} \alpha
$$

For $0<\alpha \leq \frac{1}{2} \pi$, we have $\pi-\alpha<\left(\pi-\frac{1}{2} \alpha\right) \cos \frac{1}{2} \alpha$, since $1-\frac{1}{2} \alpha^{2}<\cos \alpha$. Therefore, by $(3-3)$,

$$
Q_{\alpha}(r) \leq\left(\frac{1}{\alpha}-\frac{1}{2 \pi}\right) r, \quad 0 \leq r \leq \cos \frac{1}{2} \alpha .
$$

We also need to estimate the first derivative with respect to $r$ of $Q_{\alpha}(r)$,

$$
Q_{\alpha}^{\prime}(r)=\frac{2}{\pi \alpha} \frac{\sin \frac{1}{2} \alpha}{1+r^{2}-2 r \cos \frac{1}{2} \alpha} .
$$


It attains its maximum at the inflexion point $r=\cos \frac{1}{2} \alpha$ for $Q_{\alpha}(r)$, so that for $0<\alpha \leq \frac{1}{2} \pi$

$$
Q_{\alpha}^{\prime}(r) \leq Q_{\alpha}^{\prime}\left(\cos \frac{1}{2} \alpha\right)=\frac{2}{\pi \alpha \sin \frac{1}{2} \alpha} \leq \frac{\sqrt{2}}{\alpha^{2}}, \quad 0 \leq r \leq 1 .
$$

We use here that $(\sin x) / x \geq 2^{3 / 2} \pi^{-1}$ for $0<x \leq \pi / 4$. The function $Q_{a}^{\prime}(r)$ increases on $\left[0, \cos \frac{1}{2} \alpha\right]$, and decreases on $\left[\cos \frac{1}{2} \alpha, 1\right]$, so by estimating its values at $r=0$ and $r=1$, we see that $\left(0<\alpha \leq \frac{1}{2} \pi\right)$

$$
\begin{array}{cc}
\frac{1}{4}<Q_{\alpha}^{\prime}(r) \leq \frac{\sqrt{2}}{\alpha^{2}}, & 0 \leq r \leq 1, \\
\frac{1}{\alpha^{2}}<Q_{\alpha}^{\prime}(r) \leq \frac{\sqrt{2}}{\alpha^{2}}, & \cos \frac{1}{2} \alpha \leq r \leq 1 .
\end{array}
$$

If $A(r, \alpha)$ and $B(r, \alpha)$ stand for

$$
\begin{gathered}
A(r, \alpha)=\arctan \frac{r \sin \frac{1}{2} \alpha}{1-r \cos \frac{1}{2} \alpha}, \\
B(r, \alpha)=\left(1+r^{2}-2 r \cos \frac{1}{2} \alpha\right)^{1 / 2},
\end{gathered}
$$

then

$$
\frac{r Q_{\alpha}^{\prime}(r)}{Q_{\alpha}(r)}=\frac{\sin A(r, \alpha)}{A(r, \alpha) B(r, \alpha)} .
$$

Using this identity and the elementary estimate $2 \pi^{-1}<(\sin x) / x<1$ for $0<x<$ $\frac{\pi}{2}$, we get

$$
\begin{gathered}
\frac{1}{2}<\frac{r Q_{\alpha}^{\prime}(r)}{Q_{\alpha}(r)}, \quad 0<r \leq 1,0<\alpha \leq \frac{1}{2} \pi \\
\frac{2}{3 \alpha}<\frac{r Q_{\alpha}^{\prime}(r)}{Q_{\alpha}(r)}<\frac{3}{\alpha}, \quad \cos \frac{1}{2} \alpha \leq r \leq 1,0<\alpha \leq \frac{1}{2} \pi .
\end{gathered}
$$

At some point, we shall also need to be able to handle the function $Q_{2 \pi-\alpha}(r)$, for small positive angles $\alpha$. One shows that for $0<\alpha \leq \frac{1}{2} \pi$,

$$
\frac{1}{4}<\frac{r Q_{2 \pi-\alpha}^{\prime}(r)}{Q_{2 \pi-\alpha}(r)}<1, \quad 0<r \leq 1
$$

\section{Iteration SCheme, I}

We shall now produce an iteration scheme that produces harmonic functions with certain prescribed properties.

Lemma 4.1. Let two continuous increasing functions $u, v:[0,1[\rightarrow[1,+\infty[$ be given, with limits

$$
\begin{gathered}
\lim _{r \rightarrow 1} u(r)=\lim _{r \rightarrow 1} v(r)=+\infty, \\
\lim _{r \rightarrow 1}(1-r) u(r)=0 .
\end{gathered}
$$

Suppose that $f \in h^{\infty}(\mathbb{D})$ is a real-valued function which satisfies

$$
-v(|z|) \leq f(z) \leq u(|z|), \quad z \in \mathbb{D} .
$$


Let $\zeta_{0} \in \mathbb{T}, 0<R<1$, and $\varepsilon, N>0$ be given. Then there is a real-valued function $g \in h^{\infty}(\mathbb{D})$ and two points $z_{0}, w_{0} \in \mathbb{D}$, with $\left|z_{0}-\zeta_{0}\right|<\varepsilon$ and $\left|w_{0}-\zeta_{0}\right|<\varepsilon$, such that

$$
\begin{gathered}
|f(z)-g(z)|<\varepsilon, \quad|z| \leq R \\
-v(|z|) \leq g(z) \leq u(|z|), \quad z \in \mathbb{D},
\end{gathered}
$$

and

$$
\begin{gathered}
g\left(z_{0}\right) \geq u\left(\left|z_{0}\right|\right)-\varepsilon, \\
g\left(w_{0}\right) \leq-N .
\end{gathered}
$$

Moreover, we can get $g$ to satisfy

$$
\|f-g\|_{h^{1}}=\frac{1}{2 \pi} \int_{-\pi}^{\pi}\left|f\left(e^{i \theta}\right)-g\left(e^{i \theta}\right)\right| d \theta<\varepsilon .
$$

Proof. By replacing $f$ with $\tau f$, for some constant $\tau, 0<\tau<1$, close to 1 , we may assume that

$$
-v(|z|)<f(z)<u(|z|), \quad z \in \mathbb{D} .
$$

Moreover, by rotation invariance, we may assume $\zeta_{0}=1$.

We will construct $g$ as a suitable constant multiple of the function $h_{\lambda}$ (with appropriate choices of the real parameters $\alpha, \beta$, and $\lambda, \lambda \geq 0$ ),

$$
h_{\lambda}(z)=f(z)+\lambda \Phi_{\alpha, \beta}(z), \quad z \in \mathbb{D} .
$$

For $0<r<1$, let $D(1, r)$ be the lunula (partial moon)

$$
D(1, r)=\{z \in \mathbb{D}:|1-z| \leq r\} .
$$

For $0<\beta \leq r$, the distance from $\mathbb{D} \backslash D(1, r)$ to $I(\beta)$ is at least $r / 2$, so that by Lemma 2.1,

$$
|P \mu(z)| \leq \frac{\beta}{2 \pi} \frac{1-|z|^{2}}{d(z, I(\beta))^{3}} \leq \frac{4 \beta}{\pi r^{2}}, \quad z \in \mathbb{D} \backslash D(1, r),
$$

holds for all Borel measures $\mu$ with $\mu(\mathbb{T})=0$ and $\|\mu\| \leq 1$ that are supported on the $\operatorname{arc} I(\beta)$.

Let $0<\alpha<\beta \leq r<1$, and let $\Phi_{\alpha, \beta}$ be as before. Then the measure $\mu_{\alpha, \beta}$,

$$
d \mu_{\alpha, \beta}\left(e^{i \theta}\right)=\frac{\beta}{2(\beta-\alpha)} \Phi_{\alpha, \beta}\left(e^{i \theta}\right) d \theta,
$$

has $\mu_{\alpha, \beta}(\mathbb{T})=0,\left\|\mu_{\alpha, \beta}\right\|=1$, and it is supported on the $\operatorname{arc} I(\beta)$. Moreover,

$$
P \mu_{\alpha, \beta}(z)=\frac{\beta}{2(\beta-\alpha)} \Phi_{\alpha, \beta}(z), \quad z \in \mathbb{D},
$$

so that by (4-2), it follows that

$$
\left|\Phi_{\alpha, \beta}(z)\right| \leq 8 \pi^{-1} r^{-2}(\beta-\alpha), \quad z \in \mathbb{D} \backslash D(1, r) .
$$

If we write $\xi=8 \pi^{-1} \beta r^{-2}$, we then have, by the above estimate, (4-1), and the assumptions on $f$,

$$
\begin{gathered}
\left|h_{\lambda}(z)-f(z)\right|<\xi \lambda, \quad z \in \mathbb{D} \backslash D(1, r), \\
-v(|z|)-\xi \lambda<h_{\lambda}(z)<u(|z|)+\xi \lambda, \quad z \in \mathbb{D} \backslash D(1, r) .
\end{gathered}
$$


We clearly want the product $\xi \lambda$ to be small. Choose $\lambda, 0 \leq \lambda$, such that

$$
\sup \left\{h_{\lambda}(z)-u(|z|): z \in D(1, r)\right\}=0 ;
$$

if we rewrite this condition, we get

$$
\frac{1}{\lambda}=\sup \left\{\frac{\Phi_{\alpha, \beta}(z)}{u(|z|)-f(z)}: z \in D(1, r)\right\} .
$$

The function $u(|z|)-f(z)$ is positive throughout $\mathbb{D}$, by the argument where $f$ was replaced with $\tau f$. Since the function $u(|z|)$ tends to infinity as $|z| \rightarrow 1$, the supremum in $\left(4-4^{\prime}\right)$ is attained at some point $z_{0} \in D(1, r)$. By (4-3) and (4-4), we have

$$
\left\{\begin{array}{l}
h_{\lambda}(z) \leq u(|z|)+\xi \lambda, \quad z \in \mathbb{D}, \\
h_{\lambda}\left(z_{0}\right)=u\left(\left|z_{0}\right|\right)
\end{array}\right.
$$

so that what we now need is an estimate of $\xi \lambda$, and a corresponding estimate of $h_{\lambda}$ from below. To this end, we estimate the size of $\lambda$. Since $f \in h^{\infty}(\mathbb{D})$, and $u(t), v(t)$ both tend to $+\infty$ as $t \rightarrow 1$, we can, by taking $r$ small enough, make sure that

$$
2 N+\|f\|_{h^{\infty}}<\frac{1}{2} \min \{u(1-r), v(1-r)\}, \quad z \in D(1, r) .
$$

By (4-5) (or the less restrictive condition which results when $N$ is replaced by 0 ) and the fact that $\Phi_{\alpha, \beta}(z)>-\beta^{-1}$ throughout $\mathbb{D}$, the function $h_{\lambda}$ is estimated from below as follows:

$$
\begin{aligned}
h_{\lambda}(z) & +v(|z|)=f(z)+\lambda \Phi_{\alpha, \beta}(z)+v(|z|) \\
\geq & \frac{1}{2} v(|z|)+\lambda \Phi_{\alpha, \beta}(z)>\frac{1}{2} v(|z|)-\frac{\lambda}{\beta}, \quad z \in D(1, r) .
\end{aligned}
$$

We now fix the parameter $r$, requiring it to satisfy all the previous smallness conditions, and in addition $r<\min \{\varepsilon, 1-R\}$. This assures that the point $z_{0}$ is sufficiently close to $\zeta_{0}=1$, and that the disk $|z|<R$ is contained in $\mathbb{D} \backslash D(1, r)$. As we shall see later, given an arbitrary $\delta, 0<\delta<1$, (4-5) makes it possible to choose the parameters $\alpha, \beta$ such that the following holds:

$$
\left\{\begin{array}{c}
\max \{\lambda, \xi \lambda\}<\delta \\
2 N+\|f\|_{h^{\infty}}<\frac{\lambda}{\beta} \leq \frac{1}{2} \min \{u(1-r), v(1-r)\} .
\end{array}\right.
$$

Taking (4-7) for granted, we can now bring the proof of the lemma to its conclusion. It follows from (4-3), (4-4), (4-6), and (4-7) that $h_{\lambda}$ satisfies

$$
\begin{gathered}
\left|f(z)-h_{\lambda}(z)\right|<\delta, \quad z \in \mathbb{D} \backslash D(1, r), \\
-v(|z|)-\delta<h_{\lambda}(z)<u(|z|)+\delta, \quad z \in \mathbb{D}, \\
\inf \left\{h_{\lambda}(z): z \in D(1, r)\right\} \leq\|f\|_{h^{\infty}}-\frac{\lambda}{\beta}<-2 N .
\end{gathered}
$$

The last line implies the existence of a point $w_{0} \in D(1, r)$ with $h_{\lambda}\left(w_{0}\right)<-2 N$. Finally,

$$
\frac{1}{2 \pi} \int_{-\pi}^{\pi}\left|h_{\lambda}\left(e^{i \theta}\right)-f\left(e^{i \theta}\right)\right| d \theta=\frac{\lambda}{2 \pi} \int_{-\pi}^{\pi}\left|\Phi_{\alpha, \beta}\left(e^{i \theta}\right)\right| d \theta=\frac{\lambda}{\pi}\left(1-\frac{\alpha}{\beta}\right) \leq \frac{\lambda}{\pi}<\delta .
$$

Since we also have $h_{\lambda}\left(z_{0}\right)=u\left(\left|z_{0}\right|\right)$ and $h_{\lambda}\left(w_{0}\right)<-2 N$, the function $g(z)=\sigma h_{\lambda}(z)$ meets all the conditions of the lemma, provided $\delta$ is close to 0 , and $\sigma \in] \frac{1}{2}, 1[$ is close to 1 . 
We turn to the demonstration of (4-7). Observe that the function that we take the supremum of in $\left(4-4^{\prime}\right)$ is continuous up to the boundary of $D(1, r)$, that it is positive somewhere in $D(1, r)$, and that it depends continuously on the parameters $\alpha, \beta, \alpha<\beta$. It follows that $\lambda$ is positive, and depends continuously on $\alpha, \beta$, for $\alpha<\beta$. Write $\alpha=\vartheta \beta$, where $0<\vartheta<1$. As $\beta \rightarrow 0$ with $\vartheta$ fixed, the point $z_{0}$ where the supremum in $\left(4-4^{\prime}\right)$ is attained tends to 1 . Since the function $\beta \Phi_{\vartheta \beta, \beta}$ is bounded above (and below), and $u(t) \rightarrow+\infty$ as $t \rightarrow 1$, it follows that $\beta / \lambda \rightarrow 0$ as $\beta \rightarrow 0$, with fixed $\vartheta$. We claim that for fixed $\beta, \beta / \lambda \rightarrow+\infty$ as $\vartheta \rightarrow 0$. To this end, we note that since $0<\alpha=\vartheta \beta<r<1,1-r<1-\frac{1}{5} \vartheta \beta<1$ holds, making $1-\frac{1}{5} \vartheta \beta \in D(1, r)$. We plug in the point $z=1-\frac{1}{5} \vartheta \beta$ into the supremum in (4-4'), using (3-2) (which applies since $1-\frac{1}{2} \sin \frac{1}{2} \vartheta \beta<1-\frac{1}{5} \vartheta \beta<1$ ) and (4-5) (with $N$ replaced by 0 ); the result is, for $0<\vartheta<\frac{1}{2}$,

$$
\frac{\vartheta^{-1}-2}{3 u\left(1-\frac{1}{5} \vartheta \beta\right)} \leq \frac{\beta}{\lambda}
$$

As we let $\vartheta \rightarrow 0$, the left-hand side tends to $+\infty$, because of the growth assumption on $u$ that $(1-t) u(t) \rightarrow 0$ as $t \rightarrow 1$. The claim follows. To get (4-7), we require $\vartheta$ to be small, $0<\vartheta<\frac{1}{4}$, and note that by (4-8),

$$
0<\lambda \leq \frac{3 \vartheta \beta u\left(1-\frac{1}{5} \vartheta \beta\right)}{1-2 \vartheta} \leq 6 \vartheta \beta u\left(1-\frac{1}{5} \vartheta \beta\right) .
$$

From the above formula and the growth control of $u$ we see that we can find a $\beta_{0}$ so small that $0<\max \{\lambda, \xi \lambda\}<\delta$ for all $\beta, \vartheta, 0<\beta \leq \beta_{0}, 0<\vartheta<\frac{1}{4}$ (the parameter $\xi$ tends to 0 as $\beta \rightarrow 0$ ). Fix, for a moment, a $\vartheta \in] 0, \frac{1}{4}$ [, and note that since $\lambda / \beta \rightarrow+\infty$ as $\beta \rightarrow 0$, we can find a $\beta, 0<\beta<\beta_{0}$, such that $\lambda / \beta$ exceeds $\frac{1}{2} \max \{u(1-r), v(1-r)\}$ for the fixed value of $\vartheta$. By now letting $\vartheta$ move closer to 0 , we can reduce $\lambda / \beta$ in size, and by the intermediate value theorem, it must at some point hit the given interval. The proof is complete.

Theorem 4.2. Let two continuous increasing functions $u, v:[0,1[\rightarrow[1,+\infty[$ be given, with limits

$$
\begin{gathered}
\lim _{r \rightarrow 1} u(r)=\lim _{r \rightarrow 1} v(r)=+\infty, \\
\lim _{r \rightarrow 1}(1-r) u(r)=0 .
\end{gathered}
$$

Then there exist a real-valued function $f \in h^{1}(\mathbb{D})$, which is the Poisson integral of an $L^{1}(\mathbb{T})$ function, and a sequence $Z=\left\{z_{n}\right\}_{n}$ of points in $\mathbb{D}$ which accumulates at every point of $\mathbb{T}$, such that

$$
\begin{gathered}
-v(|z|) \leq f(z) \leq u(|z|), \quad z \in \mathbb{D}, \\
u(|z|)-1 \leq f(z), \quad z \in Z, \\
\inf \{f(z): z \in \mathbb{D}\}=-\infty .
\end{gathered}
$$

Proof. We produce iteratively functions $f_{n} \in h^{\infty}(\mathbb{D})$ and points $z_{n}, w_{n}$ in $\mathbb{D}$ as follows. We start with $f_{1}=0$. Let $\left\{\zeta_{n}\right\}_{n}$ be a dense sequence of points in $\mathbb{T}$. Suppose we have $f_{n-1}$, and points $Z_{n-1}=\left\{z_{2}, \ldots, z_{n-1}\right\}, W_{n-1}=\left\{w_{2}, \ldots, w_{n-1}\right\}$, such 
that

$$
\begin{gathered}
-v(|z|) \leq f_{n-1}(z) \leq u(|z|), \quad z \in \mathbb{D}, \\
u(|z|)-1+2^{-n+1} \leq f_{n-1}(z), \quad z \in Z_{n-1}, \\
f_{n-1}\left(w_{k}\right) \leq-k-2^{-n+1}, \quad w_{k} \in W_{n-1} .
\end{gathered}
$$

In the setting of Lemma 4.1, let $R, 0<R<1$, be bigger than any of the numbers $\left|z_{2}\right|, \ldots,\left|z_{n-1}\right|,\left|w_{2}\right|, \ldots,\left|w_{n-1}\right|$, let $\varepsilon=2^{-n}$, and let $N=n+1$. If we plug in $f_{n-1}$ in place of $f$ and $\zeta_{n}$ in place of $\zeta_{0}$ in Lemma 4.1, we get a function $f_{n}=g \in h^{\infty}(\mathbb{D})$ and points $z_{n}=z_{0} \in \mathbb{D}$ and $w_{n}=w_{0} \in \mathbb{D}$, which are both close to $\zeta_{n} \in \mathbb{T}$. The function $f_{n}$ then has

$$
\begin{gathered}
-v(|z|) \leq f_{n}(z) \leq u(|z|), \quad z \in \mathbb{D}, \\
u(|z|)-1+2^{-n} \leq f(z), \quad z \in Z_{n}=Z_{n-1} \cup\left\{z_{n}\right\}, \\
f_{n-1}\left(w_{k}\right) \leq-k-2^{-n}, \quad w_{k} \in W_{n} .
\end{gathered}
$$

Moreover, the functions $f_{n}$ in $h^{\infty}(\mathbb{D})$ form a Cauchy sequence in $h^{1}(\mathbb{D})$, so they converge to an element in $h^{1}(\mathbb{D})$ which is the Poisson integral of an $L^{1}(\mathbb{T})$ function, with all the required properties.

\section{Construction of extremally Growing functions, I}

Here we draw a conclusion from Theorem 4.2, which will be used to prove Theorem 1.2.

Corollary 5.1. Let two continuous increasing functions $u, v:[0,1[\rightarrow[1,+\infty[$ be given, with limits

$$
\begin{gathered}
\lim _{r \rightarrow 1} u(r)=\lim _{r \rightarrow 1} v(r)=+\infty, \\
\lim _{r \rightarrow 1}(1-r) u(r)=0 .
\end{gathered}
$$

Then there exist an outer function $F$ in the Nevanlinna class on $\mathbb{D}$ and a sequence $Z$ of points accumulating at every point of $\mathbb{T}$, such that

$$
\begin{gathered}
\exp (-v(|z|)) \leq F(z) \leq \exp (u(|z|)), \quad z \in \mathbb{D}, \\
\exp (u(|z|)-1) \leq F(z), \quad z \in Z, \\
\inf \{|F(z)|: z \in \mathbb{D}\}=0 .
\end{gathered}
$$

\section{ITERATION SCHEME, II}

We now describe another iteration scheme that produces harmonic functions with certain desired properties. First, however, we need some terminology. Associate to each point of $\mathbb{T}$ the standard open Privalov "ice cream" cone with a symmetric straight opening angle $\left(\frac{1}{2} \pi\right.$ radians $)$ at the point. Given a compact set $K$ in the open unit disk $\mathbb{D}$, its Privalov shadow is the set of points on the unit circle whose Privalov cones have non-empty intersection with $K$; it is a relatively open subset of $\mathbb{T}$. We say that a Borel measurable subset $E$ of $\mathbb{T}$ is $\lambda$-dense down to scale $\delta$ provided that when it is intersected with an $\operatorname{arc} L$ of length at least $\delta$, the one-dimensional Lebesgue measure of $E \cap L$ is at least $\lambda$ times that of $L$. 
Lemma 6.1. Let $u(t)=1-\log (1-t)$ and $v(t)=\beta u(t)$, for some positive real number $\beta$. Let $\lambda$ be the smaller of the two numbers $1 / 8$ and $\beta /(2 \pi)$. Suppose $f \in h^{\infty}(\mathbb{D})$ is real-valued, and that for some non-negative constants $A, B$,

$$
-v(|z|)-A \leq f(z) \leq u(|z|)+B, \quad z \in \mathbb{D} .
$$

Let $\varrho, 0<\varrho<1$, and $\varepsilon, \varepsilon>0$, be given. Then there are a radius $R$ with $\varrho<R<1$, a constant $C=C(\beta)$ only depending on $\beta$, a function $g \in h^{\infty}(\mathbb{D})$, and a compact subset $E$ of the annulus $R<|z|<1$ whose Privalov shadow is $\lambda$-dense down to scale $\varepsilon$, such that

$$
\begin{gathered}
|f(z)-g(z)| \leq \varepsilon, \quad|z| \leq \varrho, \\
-v(|z|)-A-\varepsilon \leq g(z) \leq u(|z|)+B+\varepsilon, \quad \varrho<|z|<R, \\
-v(|z|) \leq g(z) \leq u(|z|)+C, \quad R \leq|z|<1,
\end{gathered}
$$

and

$$
u(|z|)-C \leq g(z), \quad z \in E .
$$

Proof. By replacing $f(z)$ with $f(\tau z)$, for some constant $\tau, 0<\tau<1$, close to 1 , we may assume that $f$ is continuous on the closed disk $\overline{\mathbb{D}}$.

Before we proceed further with the proof, we investigate an auxiliary function $\phi_{N}$, harmonic in $\mathbb{D}$. Let $\Phi_{\alpha}$ and $Q_{\alpha}$ be as in Section 3, and for $0<\alpha<\frac{1}{2} \pi$, let $\phi_{N}(z)=\Phi_{\alpha}\left(z^{N}\right)$ and $q_{N}(r)=Q_{\alpha}\left(r^{N}\right)$, for a positive integer $N$. Then $\phi_{N}$ is harmonic in the unit disk, and $q_{N}(r)$ is the maximum of $\phi_{N}(z)$ on the circle $|z|=r$. For $k=0,1, \ldots, N-1$, let $I_{k}(\alpha, N)$ and $J_{k}(\alpha, N)$ be the arcs

$$
\begin{gathered}
I_{k}(\alpha, N)=\left\{e^{i \theta}: \theta \in\right] \frac{1}{N}\left(2 \pi k-\frac{1}{2} \alpha\right), \frac{1}{N}\left(2 \pi k+\frac{1}{2} \alpha\right)[\}, \\
J_{k}(\alpha, N)=\left\{e^{i \theta}: \theta \in\right] \frac{1}{N}\left(2 \pi k+\frac{1}{2} \alpha\right), \frac{1}{N}\left(2 \pi(k+1)-\frac{1}{2} \alpha\right)[\} .
\end{gathered}
$$

The harmonic function $\phi_{N}(z)$ extends continuously to the unit circle except for a finite set of points, and equals $1 / \alpha-1 /(2 \pi)$ on $\bigcup_{k} I_{k}(\alpha, N)$, and $-1 /(2 \pi)$ on $\bigcup_{k} J_{k}(\alpha, N)$. We shall later specify in detail how small $\alpha$ should be in relation to the parameter $\beta$ to suit our purposes. As a matter of notation, let us agree to write $\bar{I}_{k}(\alpha, N)$ and $\bar{J}_{k}(\alpha, N)$ for the closures of the respective arcs.

PART 1. For real $t$, consider the function

$$
\Lambda_{N}(t)=\sup \left\{\frac{q_{N}(r)}{u(r)-t}: \sigma(t) \leq r<1\right\}
$$

where

$$
\sigma(t)=\max \left\{\frac{1}{2}, 1-e^{-2 t}\right\} .
$$

Note that the denominator of the expression in brackets in $(6-1), u(r)-t$, is at least 1 for $\sigma(t) \leq r<1$. By the properties of the functions $u(r)$ and $\sigma(t)$, we also have that

$$
\frac{1}{2} u(r) \leq u(r)-t, \quad \sigma(t) \leq r<1 .
$$

It is clear by inspection that the supremum in (6-1) is attained at some point, because the function $u(r)$ tends to $+\infty$ as $r \rightarrow 1$. Since

$$
\frac{d}{d r} \frac{q_{N}(r)}{u(r)-t}=\frac{q_{N}^{\prime}(r)(u(r)-t)-q_{N}(r) u^{\prime}(r)}{(u(r)-t)^{2}}
$$


the left-hand side has, after some simplifications, the same sign as

$$
r^{N} \frac{Q_{\alpha}^{\prime}\left(r^{N}\right)}{Q_{\alpha}\left(r^{N}\right)}-\frac{r}{N} \frac{u^{\prime}(r)}{u(r)-t} .
$$

Suppose the variable $t$ is confined to some given finite interval $[-T, T]$. The first term of (6-3) is $>\frac{1}{2}$, by (3-7). For $r=\sigma(t)$, the second term tends to 0 as $N$ grows to infinity, so that for large $N$, the sign of (6-3) is positive, and the supremum in (6-1) is not attained at the left boundary point. So, for $N$ large, any point $r=r_{N}(t)$ where it is attained is an interior point, and hence, by elementary calculus, we have that

$$
r^{N} \frac{Q_{\alpha}^{\prime}\left(r^{N}\right)}{Q_{\alpha}\left(r^{N}\right)}=\frac{r}{N} \frac{u^{\prime}(r)}{u(r)-t}, \quad r=r_{N}(t) .
$$

This identity, together with (3-7), (3-8), and (6-2), shows that for large $N$ we can pinpoint, with a reasonable degree of accuracy, the position of a point where the maximum in (6-1) is attained,

$$
1-\frac{C_{1}}{N \log N} \leq r_{N}(t) \leq 1-\frac{C_{2}}{N \log N},
$$

where the constants $C_{1}$ and $C_{2}$ only depend on the parameter $\alpha$; we may pick $C_{1}$ to be $2 \alpha$, and $C_{2}$ to be $\alpha / 4$.

PART 2. For large $N$, put

$$
\Lambda_{N}^{*}(t)=\frac{\alpha^{-1}-(2 \pi)^{-1}}{\log (N \log N)-t} .
$$

One then calculates that

$$
\left|\frac{1}{\Lambda_{N}(t)}-\frac{1}{\Lambda_{N}^{*}(t)}\right| \leq C,
$$

for some positive constant $C=C(\alpha)$ that only depends on $\alpha$, provided $N$ is large enough. This is so because as we plug in the point $r=r_{N}(t)$ into $(6-1)$, and use the estimate (6-5), the value of the denominator on the right-hand side of (6-1) is close to $\Lambda_{N}^{*}(t)^{-1}$, and the numerator is close to 1 , because $1-r_{N}(t)^{N} \asymp(\log N)^{-1}$ and $q_{N}\left(r_{N}(t)\right)=Q_{\alpha}\left(r_{N}(t)^{N}\right)$.

By the way the parameter $\Lambda_{N}(t)$ was defined,

$$
\frac{\phi_{N}(z)}{\Lambda_{N}(t)} \leq u(|z|)-t, \quad \sigma(t) \leq|z|<1 .
$$

For large $N$, we get, by the above estimate (6-6), that the above inequality holds approximately when $\Lambda_{N}(t)$ is replaced by $\Lambda_{N}^{*}(t)$,

$$
\frac{\phi_{N}(z)}{\Lambda_{N}^{*}(t)} \leq u(|z|)-t+C, \quad \sigma(t) \leq|z|<1,
$$

with a positive constant $C$ that only depends on $\alpha$.

Put $r_{N}^{*}=1-(N \log N)^{-1}$, which is close to the points $r_{N}(t)$ where the maximum in (6-1) is attained. We shall now show that for some positive constant $C=C(\alpha)$, which only depends on $\alpha$, the following estimate holds for large $N$,

$$
u(|z|)-t-C \leq \frac{\phi_{N}(z)}{\Lambda_{N}^{*}(t)}, \quad z / r_{N}^{*} \in \bigcup_{k} \bar{I}_{k}\left(\frac{1}{2} \alpha, N\right),
$$


where $\bigcup_{k} \bar{I}_{k}\left(\frac{1}{2} \alpha, N\right)$ is the union of arcs corresponding to the smaller parameter value $\frac{1}{2} \alpha$. We first observe that by the definition of $r_{N}(t)$,

$$
\frac{\phi_{N}(z)}{\Lambda_{N}(t)}=u(|z|)-t, \quad z / r_{N}(t) \in E(N)
$$

where $E(N)$ is the set of $N$-th roots of unity (with elements $e_{k}(N)=\exp (2 \pi i k / N)$, $k=0, \ldots, N-1)$. Next, by using (3-5), we see that replacing $\Lambda_{N}(t), r_{N}(t)$ with $\Lambda_{N}^{*}(t), r_{N}^{*}$ carries the cost of introducing a positive constant $C=C(\alpha)$ depending only on $\alpha$, in the sense that

$$
u(|z|)-t-C \leq \frac{\phi_{N}(z)}{\Lambda_{N}^{*}(t)}, \quad z / r_{N}^{*} \in E(N) .
$$

Extending the estimate beyond the set $E(N)$ to the union of $\operatorname{arcs} \bigcup_{k} \bar{I}_{k}\left(\frac{1}{2} \alpha, N\right)$ requires some simple estimates of harmonic measure, which are left to the reader. The argument is simplified if one introduces the complex variable $w=z^{N}$, and recalls that $\phi_{N}(z)=\Phi_{\alpha}\left(z^{N}\right)=\Phi_{\alpha}(w)$.

PART 3. Recall that in the above, the parameter $t$ is confined to a prescribed interval $[-T, T]$, and $N$ is chosen large, depending on $T$ (and, to some extent, on $\alpha)$. We now fix $T$ to equal the supremum of $|f(z)|$ on $\mathbb{D}$. Let $e_{k}(N)=\exp (2 \pi i k / N)$ be an $N$-th root of unity, and set $t_{k}(N)=f\left(e_{k}(N)\right)$. Then $t_{k}(N)$ is confined to the interval $[-T, T]$, and we are now at liberty to consider

$$
\mu_{k}(N)=\Lambda_{N}^{*}\left(t_{k}(N)\right)^{-1}=\frac{\log (N \log N)-f\left(e_{k}(N)\right)}{\alpha^{-1}-(2 \pi)^{-1}}
$$

and the associated function

$$
\chi_{N}(z)=\frac{1}{N} \sum_{k=0}^{N-1} \mu_{k}(N) \Phi_{\alpha / N, 2 \pi / N}\left(\bar{e}_{k}(N) z\right), \quad z \in \mathbb{D} .
$$

The points $\bar{e}_{k}(N)=\exp (-2 \pi i k / N)$ are the complex conjugates of the $e_{k}(N)$. The size of the function $\Phi_{\alpha / N, 2 \pi / N}(z)$ is estimated by means of Lemma 2.1, and for large $N$, this results in $\left(0<\alpha \leq \frac{1}{2} \pi\right)$

$$
\left|\chi_{N}(z)\right| \leq \frac{\log N}{N} \frac{8 \alpha}{(1-|z|)^{2}}, \quad z \in \mathbb{D} .
$$

The function $\phi_{N}(z)$ may be written as

$$
\phi_{N}(z)=\frac{1}{N} \sum_{k=0}^{N-1} \Phi_{\alpha / N, 2 \pi / N}\left(\bar{e}_{k}(N) z\right), \quad z \in \mathbb{D},
$$

and it is now intended to compare $\chi_{N}(z)$ with the more easily analyzed function $\chi_{k, N}(z)=\mu_{k}(N) \phi_{N}(z)$. It too enjoys (for large $N$ ) the estimate

$$
\left|\chi_{k, N}(z)\right| \leq \frac{\log N}{N} \frac{8 \alpha}{(1-|z|)^{2}}, \quad z \in \mathbb{D} .
$$


The difference is

$$
\begin{aligned}
& \chi_{N}(z)-\chi_{k, N}(z)=\frac{1}{N} \sum_{j=0}^{N-1}\left(\mu_{j}(N)-\mu_{k}(N)\right) \Phi_{\alpha / N, 2 \pi / N}\left(\bar{e}_{j}(N) z\right) \\
& \quad=\left(\alpha^{-1}-(2 \pi)^{-1}\right)^{-1} \frac{1}{N} \sum_{j=0}^{N-1}\left(f\left(e_{k}(N)\right)-f\left(e_{j}(N)\right)\right) \Phi_{\alpha / N, 2 \pi / N}\left(\bar{e}_{j}(N) z\right) .
\end{aligned}
$$

By the uniform continuity of $f$ on $\overline{\mathbb{D}}$, we can fix a $\delta, \delta>0$, such that $|f(z)-f(w)| \leq$ 1 whenever $|z-w| \leq \delta$. Split up the index set $\{0,1, \ldots, N-1\}$ in two parts, with one, $X(k, N)$, consisting of those $j$ for which $\left|e_{j}(N)-e_{k}(N)\right| \leq \delta$, and the other, $Y(k, N)$, where the opposite occurs. Then, since the various building bricks $\Phi_{\alpha / N, 2 \pi / N}\left(\bar{e}_{j}(N) z\right)$ are supported on disjoint arcs of $\mathbb{T}$ for different $j$, we get that

$$
\left(\alpha^{-1}-(2 \pi)^{-1}\right)^{-1} \frac{1}{N} \sum_{j \in X(k, N)}\left|\left(f\left(e_{k}(N)\right)-f\left(e_{j}(N)\right)\right) \Phi_{\alpha / N, 2 \pi / N}\left(\bar{e}_{j}(N) z\right)\right| \leq 1 .
$$

Summing over the remaining indices in $Y(k, N)$, noticing that

$$
\left|f\left(e_{k}(N)\right)-f\left(e_{j}(N)\right)\right| \leq 2 T
$$

we obtain similarly, for $N$ so large that $2 \pi / N$ is considerably smaller than $\frac{1}{2} \delta$,

$$
\begin{array}{r}
\left(\alpha^{-1}-(2 \pi)^{-1}\right)^{-1} \frac{1}{N} \sum_{j \in Y(k, N)}\left|\left(f\left(e_{k}(N)\right)-f\left(e_{j}(N)\right)\right) \Phi_{\alpha / N, 2 \pi / N}\left(\bar{e}_{j}(N) z\right)\right| \\
\leq 2 T \omega\left(z, \mathbb{T} \backslash L_{k}, \mathbb{D}\right)
\end{array}
$$

where $\omega$ is harmonic measure, and $L_{k}=L_{k}(\delta)$ is the arc on $\mathbb{T}$ of points within distance $\frac{1}{2} \delta$ from $e_{k}(N)$. These two estimates combine to

$$
\left|\chi_{N}(z)-\chi_{k, N}(z)\right| \leq 1+2 T \omega\left(z, \mathbb{T} \backslash L_{k}, \mathbb{D}\right), \quad z \in \mathbb{D} .
$$

Let $D_{k}=D_{k}(\delta, T)$ be the intersection with $\mathbb{D}$ of a disk centered at the point $e_{k}(N)$ with radius depending only on $T, \delta$, such that $2 T \omega\left(z, \mathbb{T} \backslash L_{k}\right) \leq 1$ for $z \in D_{k}$; then, by $(6-11)$,

$$
\left|\chi_{N}(z)-\chi_{k, N}(z)\right| \leq 2, \quad z \in D_{k}
$$

There is no restriction in assuming that all the $D_{k}$ are contained in the annulus $\sigma(T)<|z|<1$, and that the radius of each $D_{k}$ is at most $\frac{1}{2} \delta$. By (6-7) and (6-8),

$$
\begin{gathered}
f\left(e_{k}(N)\right)+\chi_{k, N}(z) \leq u(|z|)+C, \quad \sigma(T) \leq|z|<1, \\
u(|z|)-C \leq f\left(e_{k}(N)\right)+\chi_{k, N}(z), \quad z / r_{N}^{*} \in \bigcup_{j} \bar{I}_{j}\left(\frac{1}{2} \alpha, N\right),
\end{gathered}
$$

so that since $\left|f(z)-f\left(e_{k}(N)\right)\right| \leq 1$ for $z \in D_{k}$, we get from (6-12) that

$$
f(z)+\chi_{N}(z) \leq f\left(e_{k}(N)\right)+\chi_{k, N}(z)+3 \leq u(|z|)+C^{\prime}, \quad z \in D_{k},
$$

and

$$
\begin{array}{r}
u(|z|)-C^{\prime} \leq f\left(e_{k}(N)\right)+\chi_{k, N}(z)-3 \leq f(z)+\chi_{N}(z), \quad \text { for } \quad z \in D_{k} \\
\text { with } z / r_{N}^{*} \in \bigcup_{j} \bar{I}_{j}\left(\frac{1}{2} \alpha, N\right),
\end{array}
$$

where $C^{\prime}=C+3$, if $C=C(\alpha)$ is the constant appearing in (6-7) and (6-8). For 
large $N$, the distance between the centers $e_{k}(N)$ of the lunuli $D_{k}$ gets much smaller than the radius (which is independent of $k$ and $N$ ), so that $\bigcup_{k} D_{k}$ contains an annulus $S<|z|<1$, where $S=S(\delta, T)$ has $0<S<1$. Moreover, for large $N$, the set $r_{N}^{*} \cup_{j} \bar{I}_{j}\left(\frac{1}{2} \alpha, N\right)$ will be contained in the annulus $S<|z|<1$, by (6-5). It hence follows from (6-13) and (6-14) that

$$
\begin{gathered}
f(z)+\chi_{N}(z) \leq u(|z|)+C^{\prime}, \quad S<|z|<1, \\
u(|z|)-C^{\prime} \leq f(z)+\chi_{N}(z), \quad z \in r_{N}^{*} \cup_{j} \bar{I}_{j}\left(\frac{1}{2} \alpha, N\right) .
\end{gathered}
$$

The set $r_{N}^{*} \cup_{j} \bar{I}_{j}\left(\frac{1}{2} \alpha, N\right)$ will play the role of $E$ in the formulation of the lemma. For large $N$, the radial projection of $E, \bigcup_{j} \bar{I}_{j}\left(\frac{1}{2} \alpha, N\right)$, will be $\lambda$-dense down to scale $\varepsilon$, with $\lambda=\alpha /(4 \pi)$, so clearly the same will be true for the Privalov shadow of $E$, which contains the radial projection as a subset.

PART 4 . Let $R_{0}$ be the bigger of the two numbers $\varrho$ and $S$. Then by (6-9), we get, for large $N$, that $\left|\chi_{N}(z)\right|<\varepsilon$ on $|z|<R_{0}$, so that the choice

$$
g(z)=f(z)+\chi_{N}(z), \quad z \in \mathbb{D},
$$

has been shown to meet all the required conditions, save the control from below. To do the remaining chore, let $R=R(\varrho, S, f)$ be such that $R_{0} \leq R<1$ and $|f(z)| \leq \frac{1}{2} \beta u(|z|)$ on $R<|z|<1$. By making $N$ a bit larger we can ensure that $\left|\chi_{N}(z)\right|<\varepsilon$ on $|z|<R$, so that if we can show that $-\chi_{N}(z) \leq \frac{1}{2} \beta u(|z|)$ on $R<|z|<1$, the function $g(z)$ will satisfy all the required conditions.

PART 5. We turn to estimating the function $\chi_{N}(z)$ from below. For $z \in D_{k}$, $\chi_{N}(z)$ is at most 2 units apart from $\chi_{k, N}(z)$, so we estimate this simpler expression instead, noting that we might as well have assumed $|f(z)|$ to have been two units smaller than what was done above, by making $R$ a tiny bit bigger. We solve the problem of estimating $-\chi_{k, N}(z) / v(|z|)=-\mu_{k}(N) \phi_{N}(z) / v(|z|)$ from above by first noting that along any concentric circle $|z|=r$, the value is the biggest when $z^{N}$ is real and negative. It is easily checked that $-\Phi_{\alpha}(-w)=\left(2 \pi \alpha^{-1}-1\right) \Phi_{2 \pi-\alpha}(w)$, and since $\phi_{N}(z)=\Phi_{\alpha}\left(z^{N}\right)$, we obtain

$$
\sup \left\{\frac{-\chi_{k, N}(z)}{v(|z|)}: z \in \mathbb{D}\right\}=\frac{2 \pi-\alpha}{\alpha \beta \Lambda_{N}^{*}\left(t_{k}(N)\right)} \sup \left\{\frac{Q_{2 \pi-\alpha}\left(r^{N}\right)}{u(r)}: 0<r<1\right\} .
$$

The extremal problem on the right-hand side is of the same kind as (6-1), and based on (3-9), one shows with the same methods as were used for problem (6-1) that the point where the above supremum is attained satisfies the analog of the estimate (6-5), only this time the constants are absolute. When this information is inserted into (6-15), one obtains, using (6-6), that for large $N$,

$$
\sup \left\{\frac{-\chi_{k, N}(z)}{v(|z|)}: z \in \mathbb{D}\right\} \leq \frac{\alpha}{4 \beta} .
$$

If we choose $\alpha$ to be the smaller of the two numbers $2 \beta$ and $\frac{1}{2} \pi$, the assertion that $-\chi_{N}(z) \leq \frac{1}{2} \beta u(|z|)$ on $R<|z|<1$ is immediate. This completes the proof of the lemma.

A relatively closed subset $E$ of $\mathbb{D}$ is said to be dominating for $H^{\infty}$ provided that

$$
\sup \{|f(z)|: z \in E\}=\sup \{|f(z)|: z \in \mathbb{D}\}=\|f\|_{H^{\infty}}
$$

holds for all $f \in H^{\infty}$. The following characterization is well known [5]: a relatively 
closed subset $E$ of $\mathbb{D}$ is dominating for $H^{\infty}$ if and only if almost every point of $\mathbb{T}$ is a non-tangential accumulation point of $E$.

Theorem 6.2. Let $u(t)=1-\log (1-t)$ and $v(t)=\beta u(t)$, for some positive real number $\beta$. Then there exist a real-valued harmonic function $f$ on $\mathbb{D}$, a positive constant $C=C(\beta)$, only depending of $\beta$, and a relatively closed subset $E$ of $\mathbb{D}$ which is dominating for $H^{\infty}$, such that

$$
\begin{gathered}
-v(|z|)-1 \leq f(z) \leq u(|z|)+C, \quad z \in \mathbb{D}, \\
u(|z|)-C \leq f(z), \quad z \in E .
\end{gathered}
$$

Proof. We produce iteratively functions $f_{n} \in h^{\infty}(\mathbb{D})$, radii $r_{n}$, and compact subsets $E_{n}$ of $\mathbb{D}$, as follows. We start with $f_{1}=0, r_{1}=\frac{1}{2}$, and $E_{1}=\emptyset$. In general, the radius $r_{n}$ will be chosen such that $r_{n-1}<r_{n}<1$, and such that the set $\bigcup_{j=1}^{n-1} E_{j}$ is contained in the disk $|z|<r_{n}$. Moreover, as $n \rightarrow+\infty$, we want $r_{n} \rightarrow 1$. Suppose we have $f_{n-1}$ and $\bigcup_{j=1}^{n-1} E_{j}$, such that

$$
\begin{gathered}
-v(|z|)-1+2^{-n+1} \leq f_{n-1}(z) \leq u(|z|)+C-2^{-n+1}, \quad z \in \mathbb{D}, \\
u(|z|)-C+2^{-n+1} \leq f(z), \quad z \in \bigcup_{j=1}^{n-1} E_{j} .
\end{gathered}
$$

Lemma 6.1, with $\varepsilon=2^{-n}$, will then deliver a compact set $E_{n}$ contained in the ring $r_{n}<|z|<1$, and a function $g=f_{n} \in h^{\infty}(\mathbb{D})$ such that

$$
\begin{gathered}
-v(|z|)-1+2^{-n} \leq f_{n}(z) \leq u(|z|)+C-2^{-n}, \quad z \in \mathbb{D}, \\
u(|z|)-C+2^{-n} \leq f_{n}(z), \quad z \in \bigcup_{j=1}^{n} E_{j} .
\end{gathered}
$$

This way we get a sequence of functions $\left\{f_{n}\right\}_{n}$ in $h^{\infty}(\mathbb{D})$, which converge, uniformly on compact subsets of $\mathbb{D}$, to a function $f$, harmonic in $\mathbb{D}$, such that

$$
\begin{gathered}
-v(|z|)-1 \leq f(z) \leq u(|z|)+C, \quad z \in \mathbb{D}, \\
u(|z|)-C \leq f(z), \quad z \in E,
\end{gathered}
$$

where $E=\bigcup_{j=1}^{\infty} E_{j}$. It remains to check that $E$ is dominating for $H^{\infty}$. To this end, it suffices to show that for each $k$, the Privalov shadow of $\bigcup_{j=k}^{\infty} E_{j}$ has full arc length measure on $\mathbb{T}$. By the construction, each $E_{j}$ has the property that its Privalov shadow is $\lambda$-dense down to scale $2^{-j}$, where $\lambda$ is a positive constant, specified in Lemma 6.1. The Privalov shadow of $\bigcup_{j=k}^{\infty} E_{j}$, which is open, then has the property that it is $\lambda$-dense down to any positive scale, no matter what $k$ is. By elementary measure theory, any such set has full measure.

\section{Construction of extremally Growing functions, II}

Here we draw a conclusion from Theorem 6.2 , which will be used to prove Theorem 1.3.

Corollary 7.1. Let $\beta>0$ be real. There exists a function $F$ which is analytic on $\mathbb{D}$ and satisfies

1. $\sup \{(1-|z|)|F(z)|: z \in \mathbb{D}\}<+\infty$,

2. $|F(z)| \geq(1-|z|)^{\beta}, \quad z \in \mathbb{D}$,

3. the set $E(F)=\{z \in \mathbb{D}:|F(z)|(1-|z|) \geq 1\}$ is dominating for $H^{\infty}$. 
Remark 7.2. The function $F$ constructed in the corollary has the additional property that

$$
\inf \{|F(z)|: z \in \mathbb{D}\}=0 \text {. }
$$

To see this, we argue as follows. If this were not the case, we would have $1 / F \in H^{\infty}$, and since bounded analytic functions possess finite radial limits almost everywhere, $F$ cannot satisfy condition 3 of the corollary.

\section{Iteration scheme, III}

Let us plunge back into the details of the construction in the proof of Lemma 6.1. We shall make some adjustments that will make it possible to produce functions of a slightly slower rate of increase.

Keep $\alpha=\min \left\{\frac{1}{2} \pi, 2 \beta\right\}$. For a fixed real parameter $\xi$, we consider in place of $t_{k}(N)=f\left(e_{k}(N)\right)$ the values $t_{k}(N, \xi)=f\left(e_{k}(N)\right)+\xi$, and hence put

$$
\mu_{k}(N, \xi)=\Lambda_{N}^{*}\left(t_{k}(N, \xi)\right)^{-1}=\frac{\log (N \log N)-t_{k}(N, \xi)}{\alpha^{-1}-(2 \pi)^{-1}},
$$

and consider the function

$$
\chi_{N, \xi}(z)=\frac{1}{N} \sum_{k=0}^{N-1} \mu_{k}(N, \xi) \Phi_{\alpha / N, 2 \pi / N}\left(\bar{e}_{k}(N) z\right), \quad z \in \mathbb{D} .
$$

For large $N$, one obtains, for some positive constant $C=C(\alpha)$, that

$$
\begin{gathered}
f(z)+\chi_{N, \xi}(z) \leq u(|z|)-\xi+C, \quad S<|z|<1, \\
u(|z|)-\xi-C \leq f(z)+\chi_{N, \xi}(z), \quad z \in r_{N}^{*} \cup_{j} I_{j}\left(\frac{1}{2} \alpha, N\right) .
\end{gathered}
$$

These considerations lead to the following rather technical lemma.

Lemma 8.1. Let $u(t)=1-\log (1-t)$ and $v(t)=\beta u(t)$, for some positive real number $\beta$. Suppose $f \in h^{\infty}(\mathbb{D})$ is real-valued, and that for some constants $A, B$, $A, B \geq 0$,

$$
-v(|z|)-A \leq f(z) \leq u(|z|)+B, \quad z \in \mathbb{D} .
$$

Let $r, 0<r<1$, and $\varepsilon, \varepsilon>0$, be given. Then, given a real parameter $\xi$, there is a radius $R$ with $r<R<1$ and a constant $C=C(\beta)$, such that for large $N$,

$$
\begin{gathered}
\quad|f(z)-g(z)| \leq \varepsilon, \quad|z| \leq r, \\
-v(|z|)-A-\varepsilon \leq g(z) \leq u(|z|)+B+\varepsilon, \quad r<|z|<R, \\
-v(|z|) \leq g(z) \leq u(|z|)-\xi+C, \quad R \leq|z|<1,
\end{gathered}
$$

and

$$
u(|z|)-\xi-C \leq g(z), \quad z \in E,
$$

where $E=r_{N}^{*} \cup_{j} \bar{I}_{j}\left(\frac{1}{2} \alpha, N\right)$, and $\alpha$ is the smaller of the two numbers $2 \beta$ and $\frac{1}{2} \pi$.

We now turn to the problem of estimating a subharmonic function $h$, about which we know a priori that it is bounded from above by some unspecified constant, and that it meets, for $n=1,2,3, \ldots$, the conditions $h(z) \leq \xi_{n}$ for $z \in E_{n}$, where $\xi_{n}$ is a sequence of real numbers tending to $+\infty$ slowly, $E_{n}$ equals $r_{N}^{*} \cup_{j} \bar{I}_{j}\left(\frac{1}{2} \alpha, N\right)$ with $N=N(n)$, and $N(n)$ is a sequence of positive integers tending to $+\infty$ rapidly. To this end, we look at the following simpler situation. 
Lemma 8.2. Let $h$ be subharmonic in a neighborhood of $\overline{\mathbb{D}}$, and let $r$ have $0<$ $r<1$. Suppose that $h(z)$ is bounded from above on $\mathbb{D}$, and that $h(z) \leq \xi$ on $\bigcup_{j} I_{j}\left(\frac{1}{2} \alpha, N\right)$. Then, if $N$ is sufficiently large, we have

$$
h(z) \leq \frac{1}{13} \alpha \xi+\left(1-\frac{1}{13} \alpha\right) M, \quad|z| \leq r,
$$

where $M=\sup \{h(z): z \in \mathbb{D}\}$.

Proof. If $M \leq \xi$ there is nothing to prove, the assertion being obvious. So, we suppose $\xi<M$, and use the maximum principle to conclude that

$$
\begin{aligned}
h(z) & \leq\left(1-\frac{\alpha}{4 \pi}\right) M+\frac{\alpha \xi}{4 \pi}-(M-\xi) \frac{1}{2} \alpha \Phi_{\alpha / 2}\left(z^{N}\right) \\
& =\left(1-\frac{\alpha}{13}\right) M+\frac{\alpha \xi}{13}-\alpha(M-\xi)\left(\frac{1}{4 \pi}-\frac{1}{13}+\frac{1}{2} \Phi_{\alpha / 2}\left(z^{N}\right)\right), \quad z \in \mathbb{D} .
\end{aligned}
$$

For $|z| \leq r$ and large $N, \Phi_{\alpha / 2}\left(z^{N}\right)$ is as small as we want, so the assertion follows from the observation that $4 \pi<13$.

Lemma 8.2 permits us to solve the above-mentioned problem.

Lemma 8.3. Let $h$ be a subharmonic function on $\mathbb{D}$, which is bounded from above, and has the estimate

$$
h(z) \leq \xi_{n}, \quad z \in r_{N(n)}^{*} \cup_{j} I_{j}\left(\frac{1}{2} \alpha, N(n)\right),
$$

for a sequence $\{N(n)\}_{n}$ of integers tending to $+\infty$, and a sequence $\left\{\xi_{n}\right\}_{n}$ of real numbers, all $\geq 0$. Then, if the $N(n)$ grow sufficiently rapidly (keeping all else fixed), we have

$$
h(z) \leq \frac{1}{13} \alpha \sum_{j=0}^{\infty}\left(1-\frac{1}{13} \alpha\right)^{j} \xi_{n+j+1}, \quad|z| \leq r_{N(n)}^{*} .
$$

Proof. Let $L$ be a constant such that $h(z) \leq L$ on $\mathbb{D}$. We will show that for $J=0,1,2,3, \ldots$,

$$
h(z) \leq \frac{1}{13} \alpha \sum_{j=0}^{J-1}\left(1-\frac{1}{13} \alpha\right)^{j} \xi_{n+j+1}+\left(1-\frac{1}{13} \alpha\right)^{J} L, \quad|z| \leq r_{N(n)}^{*} .
$$

We get (8-1) by using a backward induction argument. Clearly, (8-1) holds for $J=0$, no matter what $n$ is. Suppose (8-1) has been obtained for a particular $J$, and all $n$. Let $N(n)$ be so large that the assertion of Lemma 8.2 holds with $N=N(n), r=r_{N(n-1)}^{*} / r_{N(n)}^{*}, \xi=\xi_{n}$, and with $M$ equal to the right-hand side of (8-1) (no matter what integer $J$ is). In Lemma 8.2, $N$ grows to $\infty$ as $r$ tends to 1 , so to see that this does not concern us, note that for large $N(n)$, this $r$ is close to $r_{N(n-1)}^{*}$ which is fixed away from 1 in the construction. By that lemma and a slight dilation of the unit disk, (8-1) now holds when $J$ is replaced by $J+1$, and $n$ by $n-1$. Induction shows that estimate (8-1) holds for all $n$ and $J$.

The assertion of the lemma follows from (8-1) by letting $J$ tend to $+\infty$.

We now combine Lemmas 8.1 and 8.3. 
Theorem 8.4. Let $u$ and $v$ be as in Lemma 8.1, and $\alpha$ related to $\beta$ as before. Moreover, let $N(n)$ be an increasing sequence of positive integers tending to $+\infty$, and $E^{\cup}$ be the union over $n=1,2,3, \ldots$ of the compact sets

$$
E_{n}=r_{N(n)}^{*} \cup_{j} \bar{I}_{j}\left(\frac{1}{2} \alpha, N(n)\right)
$$

in $\mathbb{D}$. Then, if the $N(n)$ increase sufficiently rapidly, there are an increasing function $u_{0}:\left[0,1\left[\rightarrow\left[0,+\infty\left[\right.\right.\right.\right.$, with $u_{0}(t)=o(u(t))$ as $t \rightarrow 1$ and $\lim _{t \rightarrow 1} u_{0}(t)=+\infty, a$ positive constant $C=C(\alpha)$, and a function $f$, harmonic in $\mathbb{D}$, such that

$$
\begin{gathered}
-v(|z|)-1 \leq f(z) \leq u(|z|)-u_{0}(|z|)+C, \quad z \in \mathbb{D}, \\
u(|z|)-u_{0}(|z|)-C \leq f(z), \quad z \in E^{\cup}, \\
\inf \left\{f(z)+u_{0}(|z|): z \in \mathbb{D}\right\}=-\infty,
\end{gathered}
$$

and such that if a function $h$ is subharmonic and bounded from above in $\mathbb{D}$, and has $h(z) \leq u_{0}(|z|)$ for $z \in E^{\cup}$, then $h(z) \leq u_{0}(|z|)+C$ holds for all $z \in \mathbb{D}$.

Proof. The proof follows more or less the lines of that of Theorem 6.2, except the function $u_{0}$ must be defined. We produce iteratively functions $f_{n} \in h^{\infty}(\mathbb{D})$, radii $r_{n}$, and compact subsets $E_{n}^{\cup}$, as follows. Along the way, we will define increasing functions $u_{0, n}:\left[0,1\left[\rightarrow\left[0,+\infty\left[\right.\right.\right.\right.$, which tend to the desired function $u_{0}$ as $n \rightarrow+\infty$. We start with $f_{0}=0, r_{0}=\frac{1}{2}$, and $E_{1}^{\cup}=\emptyset$. We also set $u_{0,0}(t)=0$ on $[0,1[$. In general, the radius $r_{n}$ will be chosen such that $r_{n-1}<r_{n}<1$, and such that the set $E_{n-1}^{\cup}$ is contained in the disk $|z|<r_{n}$. Moreover, as $n \rightarrow+\infty$, we want $r_{n} \rightarrow 1$. Suppose we have $f_{n-1}$ and $E_{n-1}^{\cup}$, such that

$$
\begin{gathered}
-v(|z|)-1+2^{-n+1} \leq f_{n-1}(z) \leq u(|z|)-u_{0, n-1}(|z|)+C-2^{-n+1}, \quad z \in \mathbb{D}, \\
u(|z|)-u_{0, n-1}(|z|)-C+2^{-n+1} \leq f(z), \quad z \in E_{n-1}^{\cup} .
\end{gathered}
$$

If $N(n)$ is large enough, Lemma 8.1, with $\varepsilon=2^{-n}$ and $\xi=\xi_{n}=n$, will then deliver a radius $R=R_{n}$ with $r_{n}<R_{n}<1$, a compact set

$$
E_{n}=r_{N(n)}^{*} \cup_{j} \bar{I}_{j}\left(\frac{1}{2} \alpha, N(n)\right)
$$

contained in the ring $R_{n}<|z|<1$, and a function $g=f_{n} \in h^{\infty}(\mathbb{D})$ such that (use a slightly different constant than in the lemma)

$$
\begin{gathered}
-v(|z|)-1+2^{-n} \leq f_{n}(z) \leq u(|z|)-u_{0, n-1}(|z|)+C-2^{-n}, \quad|z|<R_{n}, \\
-v(|z|) \leq f_{n}(z) \leq u(|z|)-n-1+C, \quad R_{n} \leq|z|<1, \\
u(|z|)-u_{0, n-1}(|z|)-C+2^{-n} \leq f_{n}(z), \quad z \in E_{n-1}^{\cup}, \\
u(|z|)-n-C+1 \leq f_{n}(z), \quad z \in E_{n} .
\end{gathered}
$$

Declare $u_{0, n}(t)$ to be $u_{0, n-1}(t)$ for $0 \leq t<R_{n}$, and $n$ for $R_{n} \leq t<1$. It is readily checked that this defines an increasing function. Also, put $E_{n}^{\cup}=E_{n-1}^{\cup} \cup E_{n}$. The above estimates then simplify,

$$
\begin{gathered}
-v(|z|)-1+2^{-n} \leq f_{n}(z) \leq u(|z|)-u_{0, n}(|z|)+C-2^{-n}, \quad z \in \mathbb{D}, \\
u(|z|)-u_{0, n}(|z|)-C+2^{-n} \leq f_{n}(z), \quad z \in E_{n}^{\cup} .
\end{gathered}
$$

As $n \rightarrow+\infty$, the functions $f_{n} \in h^{\infty}(\mathbb{D})$ converge, uniformly on compact subsets of $\mathbb{D}$, to a function $f$, harmonic in $\mathbb{D}$, and the functions $u_{0, n}$ converge to a function 
$u_{0}$. These two limit functions enjoy the estimate

$$
\begin{gathered}
-v(|z|)-1 \leq f(z) \leq u(|z|)-u_{0}(|z|)+C, \quad z \in \mathbb{D}, \\
u(|z|)-u_{0}(|z|)-C \leq f(z), \quad z \in E^{\cup},
\end{gathered}
$$

where $E^{\cup}=\bigcup_{j=1}^{\infty} E_{j}$. For rapidly increasing $N(n)$, the $R(n)$, being contained between $r_{N(n-1)}^{*}$ and $r_{N(n)}^{*}$, tend to 1 very rapidly in $n$, so that we can make $u_{0}(t)$ go to $+\infty$ as slowly as we like as $t \rightarrow 1$. In particular, we can get $u_{0}(t)=o(u(t))$ as $t \rightarrow 1$.

We now turn to the assertion that $f(z)+u_{0}(|z|)$ is unbounded from below on $\mathbb{D}$. Since $u_{0}(t)=o(u(t))$ as $t \rightarrow 1$, we have, for $z \in E_{\cup}$ with $|z|$ close to 1 , that $\frac{1}{2} u(|z|) \leq f(z)$. By the mean value property of harmonic functions, it follows that $f(z)$ must be big negative for many $z$ on $r_{N(n)}^{*} \mathbb{T}$, for each $n$, and the order of magnitude is a negative constant times $u(|z|)$. It follows that $f(z)+u_{0}(|z|)$ cannot be bounded from below.

Finally, we turn to the assertion that a subharmonic function $h$ on $\mathbb{D}$ which is bounded from above and has $h(z) \leq u_{0}(|z|)$ satisfies $h(z) \leq u_{0}(|z|)+C$ throughout $\mathbb{D}$. To this end, we apply Lemma 8.3 , with $\xi_{n}=n$, and conclude that

$$
\begin{aligned}
h(z) \leq \frac{1}{13} \alpha \sum_{j=0}^{\infty} & \left(1-\frac{1}{13} \alpha\right)^{j}(n+j+1) \\
& =n+13 \alpha^{-1} \leq u_{0}(|z|)+1+13 \alpha^{-1}, \quad r_{N(n-1)}^{*}<|z| \leq r_{N(n)}^{*} .
\end{aligned}
$$

The proof is complete.

\section{Construction of extremally Growing functions, III}

Here we draw a conclusion from Theorem 8.4, which will be used to prove Theorem 1.1.

Corollary 9.1. Let $\beta>0$ be real. Then there are an increasing function $u_{0}$ : $\left[0,1\left[\rightarrow\left[0,+\infty\left[\right.\right.\right.\right.$, with $u_{0}(t)=o(-\log (1-t))$ as $t \rightarrow 1$ and $\lim _{t \rightarrow 1} u_{0}(t)=+\infty$, a function $F$ holomorphic on $\mathbb{D}$, and a relatively closed subset $E$ of $\mathbb{D}$, such that

1. $|F(z)| \leq C(1-|z|)^{-1} \exp \left(-u_{0}(|z|)\right)$ on $\mathbb{D}$, for some positive constant $C$,

2. $\quad(1-|z|)^{\beta} \leq|F(z)|$ for all $z \in \mathbb{D}$,

3. $(1-|z|)^{-1} \exp \left(-u_{0}(|z|)\right) \leq|F(z)|$ for all $z \in E$,

4. $\quad \inf \left\{\exp \left(u_{0}(|z|)\right)|F(z)|: z \in \mathbb{D}\right\}=0$, and

5. if $h$ is subharmonic and bounded from above on $\mathbb{D}$, and has $h(z) \leq u_{0}(|z|)$ on $E$, then $h(z) \leq u_{0}(|z|)+C$ on $\mathbb{D}$, with a constant $C$ that does not depend on $h$.

\section{Iteration SCHEME, IV}

We return to the setting of Section 8. Our first observation is that the assertion of Lemma 8.1 remains valid if the set $E$ is replaced by $E^{\sharp}$, where

$$
E^{\sharp}=\left\{r e^{i \theta}: r_{N}^{\sharp} \leq r \leq r_{N}^{*}, \quad e^{i \theta} \in \bigcup_{j} \bar{I}_{j}\left(\frac{1}{2} \alpha, N\right)\right\},
$$

and $r_{N}^{\sharp}=1-2(N \log N)^{-1}$. We need to have an additional estimate of the function $g=f+\chi_{N, \xi}$ produced in Lemma 8.1, which is so accurate that it allows us to say 
how big the integral

$$
\int_{\mathbb{D}} \exp (g(z)) d S(z)
$$

is. To this end, we look again at the extremal problem in (6-1), for $t=0$, this time for parameter values $\frac{1}{2} \leq r \leq\left(\cos \frac{1}{2} \alpha\right)^{1 / N}$, and note by the considerations involving the sign of (6-3) that the extremal value is attained at the right end point $r=\left(\cos \frac{1}{2} \alpha\right)^{1 / N}$, at least for large $N$. This entails that

$$
\begin{aligned}
q_{N}(r) \leq \frac{q_{N}\left(\left(\cos \frac{1}{2} \alpha\right)^{1 / N}\right)}{u\left(\left(\cos \frac{1}{2} \alpha\right)^{1 / N}\right)} u(r) & =\frac{Q_{\alpha}\left(\cos \frac{1}{2} \alpha\right)}{u\left(\left(\cos \frac{1}{2} \alpha\right)^{1 / N}\right)} u(r)=\frac{\alpha^{-1}-\pi^{-1}}{u\left(\left(\cos \frac{1}{2} \alpha\right)^{1 / N}\right)} u(r) \\
\leq & \frac{\alpha^{-1}-\pi^{-1}}{\log N+C(\alpha)} u(r), \quad \frac{1}{2} \leq r \leq\left(\cos \frac{1}{2} \alpha\right)^{1 / N},
\end{aligned}
$$

where $C(\alpha)$ is a real-valued constant. In the setting of the estimate (6-13), with the necessary modifications due to $\xi$, we then arrive (for big $N$ ) at

$$
\begin{aligned}
& f\left(e_{k}(N)\right)+\mu_{k}(N, \xi) \phi_{N}(z) \leq f\left(e_{k}(N)\right)+\mu_{k}(N, \xi) q_{N}(|z|) \\
& \quad \leq f\left(e_{k}(N)\right)+\left(1-\frac{\alpha}{2 \pi}\right) u(|z|), \quad \frac{1}{2} \leq|z| \leq\left(\cos \frac{1}{2} \alpha\right)^{1 / N} .
\end{aligned}
$$

For $R=R(r, S, f, \xi, \alpha), R_{0} \leq R<1$ (notation as in the proof of Lemma 6.1), sufficiently close to 1 , any fixed fraction of $u(|z|)$ will come to dominate over $f\left(e_{k}(N)\right)+\xi$ for $R \leq|z|<1$, so that we can get

$$
f\left(e_{k}(N)\right)+\mu_{k}(N, \xi) \phi_{N}(z) \leq\left(1-\frac{\alpha}{8}\right) u(|z|)-\xi, \quad R \leq|z| \leq\left(\cos \frac{1}{2} \alpha\right)^{1 / N} .
$$

By (10-2) and the appropriate analog of (6-13) involving $\xi$, it follows that

$$
f(z)+\chi_{N, \xi}(z) \leq\left(1-\frac{\alpha}{8}\right) u(|z|)-\xi+3, \quad R \leq|z| \leq\left(\cos \frac{1}{2} \alpha\right)^{1 / N} .
$$

By jacking up $N$ further, we can ensure that $\left|\chi_{N, \xi}(z)\right|<\varepsilon$ holds on $|z| \leq R$. To control $f+\chi_{N, \xi}$ in the remaining annulus, we first need some elementary estimates of $Q_{\alpha}$, namely, that for $r_{0}, \cos \frac{1}{2} \alpha<r_{0}<1$, the following holds, by (3-6),

$$
\begin{array}{cc}
Q_{\alpha}(r)=Q_{\alpha}\left(r_{0}\right)-\int_{r}^{r_{0}} Q_{\alpha}^{\prime}(t) d t \leq Q_{\alpha}\left(r_{0}\right)-\alpha^{-2}\left(r_{0}-r\right), \quad \cos \frac{1}{2} \alpha \leq r \leq r_{0}, \\
Q_{\alpha}(r)=Q_{\alpha}\left(r_{0}\right)+\int_{r_{0}}^{r} Q_{\alpha}^{\prime}(t) d t \leq Q_{\alpha}\left(r_{0}\right)+\sqrt{2} \alpha^{-2}\left(r-r_{0}\right), \quad r_{0} \leq r \leq 1 .
\end{array}
$$

With $r_{0}=\left(r_{N}^{*}\right)^{N}$, these estimates lead to

$$
\begin{gathered}
q_{N}(r) \leq q_{N}\left(r_{N}^{*}\right)-\alpha^{-2}\left(\left(r_{N}^{*}\right)^{N}-r^{N}\right), \quad\left(\cos \frac{1}{2} \alpha\right)^{1 / N} \leq r \leq r_{N}^{*}, \\
q_{N}(r) \leq q_{N}\left(r_{N}^{*}\right)+\sqrt{2} \alpha^{-2}\left(r^{N}-\left(r_{N}^{*}\right)^{N}\right), \quad r_{N}^{*} \leq r \leq 1 .
\end{gathered}
$$

For large $N$, we know that

$$
f\left(e_{k}(N)\right)+\mu_{k}(N, \xi) q_{N}\left(r_{N}^{*}\right)
$$


is within an additive constant (depending only on $\alpha$ ) from $u\left(r_{N}^{*}\right)-\xi=1+$ $\log (N \log N)-\xi$ (look at how we got (6-13) and (6-14)). Using the above estimates of $q_{N}(r)$, we arrive (for large $N$ ) at

$$
\begin{aligned}
& f\left(e_{k}(N)\right)+\mu_{k}(N, \xi) \phi_{N}(z) \leq f\left(e_{k}(N)\right)+\mu_{k}(N, \xi) q_{N}(|z|) \\
\leq & u\left(r_{N}^{*}\right)-\xi-\frac{\log N}{\alpha(1-\alpha /(2 \pi))}\left(1-|z|^{N}\right)+C(\alpha), \quad\left(\cos \frac{1}{2} \alpha\right)^{1 / N} \leq|z| \leq r_{N}^{*},
\end{aligned}
$$

and

$$
\begin{aligned}
f\left(e_{k}(N)\right)+\mu_{k}(N, \xi) \phi_{N}(z) \leq f\left(e_{k}(N)\right) & +\mu_{k}(N, \xi) q_{N}(|z|) \\
& \leq u\left(r_{N}^{*}\right)-\xi+C(\alpha), \quad r_{N}^{*} \leq|z|<1,
\end{aligned}
$$

for some constant $C(\alpha)$. By the localization trick of (6-13), these estimates lead to

$$
\begin{array}{r}
f(z)+\chi_{N, \xi}(z) \leq u\left(r_{N}^{*}\right)-\frac{\log N}{\alpha(1-\alpha /(2 \pi))}\left(1-|z|^{N}\right)-\xi+C^{\prime}(\alpha), \\
\left(\cos \frac{1}{2} \alpha\right)^{1 / N} \leq|z| \leq r_{N}^{*},
\end{array}
$$

and

$$
f(z)+\chi_{N, \xi}(z) \leq u\left(r_{N}^{*}\right)-\xi+C^{\prime}(\alpha), \quad r_{N}^{*} \leq|z|<1,
$$

for some other constant $C^{\prime}(\alpha)$. It follows from (10-5) and $u\left(r_{N}^{*}\right)=1+\log (N \log N)$ that

$$
\int_{r_{N}^{*} \leq|z|<1} \exp \left(f(z)+\chi_{N, \xi}(z)\right) d S(z) \leq C(\alpha) e^{-\xi},
$$

where $C(\alpha)$ is a positive constant. An exercise involving Taylor series shows that for positive real $\tau$,

$$
\int_{\mathbb{D}} \exp \left(-\tau\left(1-|z|^{N}\right)\right) d S(z) \leq e^{-\tau}+\frac{4}{N} \frac{1-e^{-\tau}}{\tau},
$$

so that by (10-4), we have, provided that $\alpha$ is not too big, that is, $\alpha(1-\alpha /(2 \pi))<1$,

$$
\int_{\left(\cos \frac{1}{2} \alpha\right)^{1 / N}<|z|<r_{N}^{*}} \exp \left(f(z)+\chi_{N, \xi}(z)\right) d S(z) \leq C(\alpha) e^{-\xi},
$$

where $C(\alpha)$ is a positive constant, possibly different from the earlier one. By (10-3), we get, since $1-\alpha / 8<1$,

$$
\int_{R<|z|<\left(\cos \frac{1}{2} \alpha\right)^{1 / N}} \exp \left(f(z)+\chi_{N, \xi}(z)\right) d S(z) \leq C(\alpha) e^{-\xi},
$$

where $C(\alpha)$ is yet another positive constant. Moreover, since $\left|\chi_{N, \xi}(z)\right|<\varepsilon$ on $|z|<R$, we obtain

$$
\int_{|z|<R} \exp \left(f(z)+\chi_{N, \xi}(z)\right) d S(z) \leq e^{\varepsilon} \int_{\mathbb{D}} \exp (f(z)) d S(z) .
$$

The above estimates (10-6) through (10-9) show that the following refinement of Lemma 8.1 is valid. One last thing: the assertion is formulated in such a way that the initial dilation, replacing $f(z)$ with $f(\tau z)$, for some $\tau, 0<\tau<1$, very close to 1 , is permissible. 
Lemma 10.1. Let all parameters and functions be as in Lemma 8.1, with the exception that $\alpha$ should be a little smaller: $\alpha=\min \{1,2 \beta\}$. Then the function $g$ can be assumed to meet all the requirements specified in Lemma 8.1, with E replaced by $E^{\sharp}$, and the additional condition that

$$
\int_{\mathbb{D}} \exp (g(z)) d S(z) \leq C e^{-\xi}+e^{\varepsilon} \int_{\mathbb{D}} \exp (f(z)) d S(z),
$$

for some positive constant $C=C(\beta)$.

As in Section 8, our next job is to handle the following problem. Let $N(n)$ be a sequence of positive integers approaching $+\infty$ rapidly, and let $E_{n}^{\sharp}$ be given by (10-1), with $N=N(n)$. Moreover, let $\xi_{n}$ be a sequence of real numbers greater than or equal to 0 , which tend to $+\infty$ rather slowly. For a Borel subset $E$ of $\mathbb{D}$, let $S(E)$ denote the normalized area of $E$ (smaller than the usual area by a factor of $\pi^{-1}$ ), and if instead $E$ is a rectifiable curve or a (relative Borel) subset of one, let $s(E)$ be the length of $E$, normalized by the factor $(2 \pi)^{-1}$. The latter definition is related to $d s$, normalized one-dimensional Lebesgue measure in the complex plane. Suppose $h$ is a subharmonic function, about which it is known that it is bounded from above by some unspecified constant, that

$$
h(z) \leq \gamma u(|z|), \quad z \in \mathbb{D},
$$

for some positive constant $\gamma$, and that

$$
\sum_{n=1}^{\infty} e^{-\xi_{n}} \frac{1}{S\left(E_{n}^{\sharp}\right)} \int_{E_{n}^{\sharp}} \exp (h(z)) d S(z) \leq A<+\infty .
$$

We wish to estimate the average radial growth of $h(z)$; to be more to the point, we want to know how quickly the integral mean

$$
\int_{-\pi}^{\pi} h^{+}\left(r e^{i \theta}\right) d \theta
$$

increases as $r$ approaches 1 , where $h^{+}(z)=\max \{h(z), 0\}$. Fubini's theorem tells us that there is a radius $\rho_{n}, r_{N(n)}^{\sharp}<\rho_{n}<r_{N(n)}^{*}$, such that with $E_{n}=E_{n}^{\sharp} \cap \rho_{n} \mathbb{T}$,

$$
\frac{1}{s\left(E_{n}\right)} \int_{E_{n}} \exp (h(z)) d s(z) \leq \frac{1}{S\left(E_{n}\right)} \int_{E_{n}^{\sharp}} \exp (h(z)) d S(z),
$$

so that

$$
\sum_{n=1}^{\infty} e^{-\xi_{n}} \frac{1}{s\left(E_{n}\right)} \int_{E_{n}} \exp (h(z)) d s(z) \leq A<+\infty .
$$

A crude estimate of each term leads to

$$
\frac{1}{s\left(E_{n}\right)} \int_{E_{n}} \exp (h(z)) d s(z) \leq A e^{\xi_{n}}
$$

We note that $E_{n}=\rho_{n} \cup_{j} \bar{I}_{j}\left(\frac{1}{2} \alpha, N(n)\right)$, so that $s\left(E_{n}\right)=\alpha \rho_{n} /(4 \pi)$ tends to $\alpha /(4 \pi)$ as $n \rightarrow+\infty$. Introduce the union of rectangular boxes $\Sigma_{n}$,

$$
\Sigma_{n}=\left\{r e^{i \theta}: \rho_{n} \leq r<1, \quad e^{i \theta} \in \bigcup_{j} \bar{I}_{j}\left(\frac{1}{4} \alpha, N(n)\right)\right\},
$$

and put a hat on each box to form $\Pi_{n}=E_{n} \cup \Sigma_{n}$. The set $\Pi_{n}$ looks like a collection of identical mushrooms, with stems affixed to the ground, the unit circle. Let $\Omega_{n}=\mathbb{D} \backslash \bigcup_{j=n}^{\infty} \Pi_{j}$, which is an open subset of $\mathbb{D}$, possibly having many components, and let $\Omega_{n}^{\natural}$ be the connectivity component that contains the origin (see Figure 10-1 


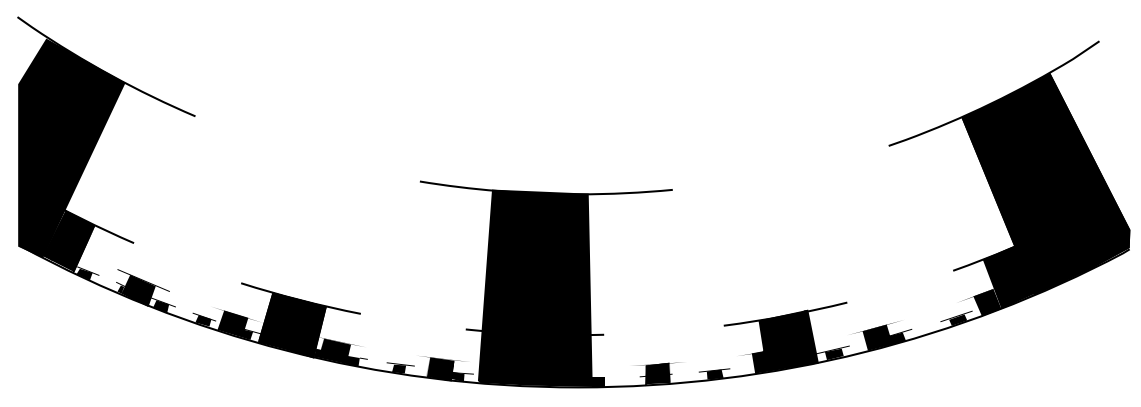

FiguRE 10-1

for a graphic illustration of the set $\Omega_{n}^{\natural}$ ), which is easily seen to be simply connected. The boundary $\partial \Omega_{n}^{\natural}$ of $\Omega_{n}^{\natural}$ consists of a closed subset of the unit circle $\mathbb{T}$, mushroom hats $E_{j}$, or parts of them, and stem sides

$$
\partial^{b} \Sigma_{j}=\left\{r e^{i \theta}: \rho_{j} \leq r<1, \quad e^{i \theta} \in \bigcup_{j} \partial I_{j}\left(\frac{1}{4} \alpha, N(n)\right)\right\},
$$

or parts of them, as well, for $j=n, n+1, \ldots$; on the right-hand side of the displayed formula, the $\partial$ is the boundary operation with respect to the topology of $\mathbb{T}$. Recall that we reserve the symbol $\omega$ for harmonic measure; we will sometimes write $d \omega(z, \zeta, \Omega)$, and think of it as a measure, where the variable of integration is $\zeta$. Since $h(z)$ is subharmonic and bounded above in $\mathbb{D}, h^{+}(z)$ is subharmonic too, and bounded from above and below. If $h_{n}$ is the function harmonic in $\Omega_{n}^{\natural}$ defined as

$$
h_{n}(z)=\int_{\partial \Omega_{n}^{\natural}} h^{+}(\zeta) d \omega\left(z, \zeta, \Omega_{n}^{\natural}\right), \quad z \in \Omega_{n}^{\natural},
$$

then, by the maximum principle, $h^{+}(z) \leq h_{n}(z)$ on $\Omega_{n}^{\natural}$. For $r$ such that $r \overline{\mathbb{D}} \subset \Omega_{n}^{\natural}$, by the mean value property for harmonic functions we have

$$
\frac{1}{r} \int_{r \mathbb{T}} h^{+}(z) d s(z) \leq \frac{1}{r} \int_{r \mathbb{T}} h_{n}(z) d s(z)=h_{n}(0)=\int_{\partial \Omega_{n}^{\natural}} h^{+}(\zeta) d \omega\left(0, \zeta, \Omega_{n}^{\natural}\right) .
$$

This calculation leaves us with the desire to estimate $\omega\left(0, L, \Omega_{n}^{\natural}\right)$ for various Borel subsets $L$ of $\partial \Omega_{n}^{\natural}$. One quickly checks it is 0 if $L$ is a subset of $\mathbb{T} \cap \partial \Omega_{n}^{\natural}$. The principle of extension of the domain states that the harmonic measure of a piece of the boundary of a region with respect to a fixed interior point gets larger if the region is expanded in such a way that the boundary piece remains on the boundary. If $L$ is a Borel subset of $Y_{j, k}=\rho_{k} \bar{I}_{j}\left(\frac{1}{2} \alpha, N(k)\right) \subset E_{k} \cap \partial \Omega_{n}^{\natural}$, for some $k=n, n+1, \ldots$, we replace $\Omega_{n}^{\natural}$ with $\mathbb{D} \backslash Y_{j, k}$, and see that

$$
\omega\left(0, L, \Omega_{n}^{\natural}\right) \leq \omega\left(0, L, \mathbb{D} \backslash Y_{j, k}\right) \leq C(\alpha) s(L),
$$

for some positive constant $C(\alpha)$. The remaining type of boundary parts is formed by the stem sides. So, let $L$ be a subset of $\partial^{b} \Sigma_{k} \cap \partial \Omega_{n}^{\natural}$ for some $k=n, n+1, \ldots$, and suppose for simplicity that it is a subset of a single stem side of one mushroom. Then, if we remove all the other mushrooms, the harmonic measure of $L$ increases, but it is still quite small, because if we think of harmonic measure as arising from Brownian motion, to reach $L$, the particle has first to reach some point of the opening between the hat and the unit circle, then it must also hit the stem, and in particular, the part that lies on $L$. The hat and the stem define a "boxed" region 
of dimension $\frac{1}{8} \alpha N(k)^{-1}$ by $1-\rho_{k}$, so that an estimate of the second process using harmonic measure for the boxed region shows that

$$
\omega\left(0, L, \Omega_{n}^{\natural}\right) \leq C(\alpha) N(k)^{-\nu(\alpha)} s(L),
$$

for some positive constants $C(\alpha), \nu(\alpha) ; \nu(\alpha)=\alpha / 6$ should do. The estimates (10-12) and (10-13) will be used to control

$$
\int_{\partial \Omega_{n}^{\natural}} h^{+}(\zeta) d \omega\left(0, \zeta, \Omega_{n}^{\natural}\right)
$$

on the hats of the mushrooms, and (10-10) and (10-14) to control it on the stems. Not all mushrooms are so lucky as to form part of the boundary of $\Omega_{n}^{\natural}$, as many are contained in the stems of earlier generations of them, and some are trapped between two bigger intersecting mushrooms. Approximately the proportion $\alpha /(8 \pi)$ of those remaining are lost with each new generation, and by jacking up the growth of the $N(n)$, we may safely claim that the proportion is between $\alpha / 30$ and $\alpha / 20$ each time.

We first do the stems. In generation $k(k=n, n+1, \ldots)$, there are $N(k)$ different mushrooms in $\Pi_{k}$, but at most $(1-\alpha / 30)^{k-n} N(k)$ of them make it to $\partial \Omega_{n}^{\natural}$. The integral of $u(|z|)$ along the two sides of a single mushroom is at most

$$
2 \int_{\rho_{k}}^{1} u(t) d t \leq \frac{6}{N(k)}
$$

where the estimate holds for large $N(k)$. It follows from (10-10) and (10-14) that the integral (10-15) taken only over the stems is bounded by the series

$$
C(\alpha) \gamma \sum_{k=n}^{\infty}\left(1-\frac{\alpha}{30}\right)^{k-n} N(k)^{-\nu(\alpha)},
$$

which converges quite fast.

We turn to the hats. In generation $k(k=n, n+1, \ldots)$, there are $N(k)$ different mushrooms in $\Pi_{k}$, but at most $(1-\alpha / 30)^{k-n} N(k)$, and at least $(1-\alpha / 20)^{k-n} N(k)$, of them make it to $\partial \Omega_{n}^{\natural}$. Since $\exp \left(h^{+}\right) \leq \exp (h)+1$, an application of Jensen's inequality shows that

$$
\begin{aligned}
& \frac{1}{s\left(E_{k} \cap \partial \Omega_{n}^{\natural}\right)} \int_{E_{k} \cap \partial \Omega_{n}^{\natural}} h^{+}(z) d s(z) \\
& \quad \leq \log \left(1+\frac{1}{s\left(E_{k} \cap \partial \Omega_{n}^{\natural}\right)} \int_{E_{k} \cap \partial \Omega_{n}^{\natural}} \exp (h(z)) d s(z)\right),
\end{aligned}
$$

and together with (10-12) and the fact that

$$
\left(1-\frac{\alpha}{20}\right)^{k-n} \leq \frac{s\left(E_{k} \cap \partial \Omega_{n}^{\natural}\right)}{s\left(E_{k}\right)} \leq\left(1-\frac{\alpha}{30}\right)^{k-n},
$$

it shows that

$$
\int_{E_{k} \cap \partial \Omega_{n}^{\natural}} h^{+}(z) d s(z) \leq\left(1-\frac{\alpha}{30}\right)^{k-n} s\left(E_{k}\right) \log \left(1+\left(1-\frac{\alpha}{20}\right)^{n-k} A e^{\xi_{k}}\right) .
$$


Since $s\left(E_{k}\right)=\alpha \rho_{k} /(4 \pi)<\alpha /(4 \pi)$, it follows that

$$
\begin{aligned}
& \sum_{k=n}^{\infty} \int_{E_{k} \cap \partial \Omega_{n}^{\natural}} h^{+}(z) d s(z) \\
& \quad \leq \frac{\alpha}{4 \pi} \sum_{k=n}^{\infty}\left(1-\frac{\alpha}{30}\right)^{k-n} \log \left(1+\left(1-\frac{\alpha}{20}\right)^{n-k} A e^{\xi_{k}}\right),
\end{aligned}
$$

where the right-hand side converges, provided

$$
\sum_{k=1}^{\infty}\left(1-\frac{\alpha}{30}\right)^{k} \xi_{k}<+\infty
$$

By the estimates (10-13) and (10-16), the integral (10-15) is controlled on the hats as well. For the choice $\xi_{n}=2 \log n$, we get more specifically

$$
\frac{1}{r} \int_{r \mathbb{T}} h^{+}(z) d s(z) \leq \int_{\partial \Omega_{n}^{\natural}} h^{+}(\zeta) d \omega\left(0, \zeta, \Omega_{n}^{\natural}\right) \leq C+C^{\prime} \xi_{n}=C+2 C^{\prime} \log n,
$$

where $C=C(\alpha, \gamma, A)$ and $C^{\prime}=C^{\prime}(\alpha)$ are positive constants. We formulate this as a lemma.

Lemma 10.2. Let $h$ be a subharmonic function on $\mathbb{D}$ which is bounded above, and write $\xi_{n}=2 \log n$. Suppose $h$ satisfies, for positive constants $\gamma, A$,

$$
h(z) \leq \gamma u(|z|), \quad z \in \mathbb{D},
$$

and

$$
\sum_{n=1}^{\infty} e^{-\xi_{n}} \frac{1}{S\left(E_{n}^{\sharp}\right)} \int_{E_{n}^{\sharp}} \exp (h(z)) d S(z) \leq A .
$$

Then if the sequence $N(n)$ increases sufficiently rapidly, there are two positive constants $C=C(\alpha, \gamma, A)$ and $C^{\prime}=C^{\prime}(\alpha)$ such that the following estimate holds, for $n=1,2,3, \ldots$ :

$$
\frac{1}{r} \int_{r \mathbb{T}} h^{+}(z) d s(z) \leq C+C^{\prime} \xi_{n}, \quad 0<r<\rho_{n}
$$

We now combine Lemmas 10.1 and 10.2.

Theorem 10.3. Let $u$ and $v$ be as in Lemma 8.1, and $\alpha=\min \{1,2 \beta\}$. Moreover, let $N(n)$ be an increasing sequence of positive integers approaching $+\infty$, and $E^{\sharp, \cup}$ be the union over $n=1,2,3, \ldots$ of the sets

$$
E_{n}^{\sharp}=\left\{r e^{i \theta}: r_{N(n)}^{\sharp} \leq r \leq r_{N(n)}^{*}, \quad e^{i \theta} \in \bigcup_{j} \bar{I}_{j}\left(\frac{1}{2} \alpha, N(n)\right)\right\},
$$

where

$$
r_{N}^{\sharp}=1-2(N \log N)^{-1} \quad \text { and } \quad r_{N}^{*}=1-(N \log N)^{-1} \text {. }
$$

Then, if the $N(n)$ increase sufficiently rapidly, there are an increasing function $u_{0}:\left[0,1\left[\rightarrow\left[0,+\infty\left[\right.\right.\right.\right.$, with $u_{0}(t)=o(u(t))$ as $t \rightarrow 1$ and $\lim _{t \rightarrow 1} u_{0}(t)=+\infty, a$ positive constant $C=C(\alpha)$, and a function $f$, harmonic in $\mathbb{D}$, such that with the 
notation $f^{-}(z)=\max \{-f(z), 0\}$,

$$
\begin{gathered}
-v(|z|)-1 \leq f(z) \leq u(|z|)-u_{0}(|z|)+C, \quad z \in \mathbb{D}, \\
u(|z|)-u_{0}(|z|)-C \leq f(z), \quad z \in E^{\sharp, \cup,} \\
\int_{\mathbb{D}} \exp (f(z)) d S(z) \leq C, \\
\limsup _{r \rightarrow 1}\left(\frac{1}{r} \int_{r \mathbb{T}} f^{-}(z) d s(z)-\lambda u_{0}(r)\right)=+\infty,
\end{gathered}
$$

for each real $\lambda, 0<\lambda$, and such that if $h$ is subharmonic and bounded above on $\mathbb{D}$, and

$$
\int_{\mathbb{D}} \exp (f(z)+h(z)) d S(z) \leq 1
$$

then

$$
\frac{1}{r} \int_{r \mathbb{T}} h^{+}(z) d s(z) \leq C\left(1+u_{0}(r)\right), \quad 0<r<1 .
$$

Proof. We proceed as in the earlier proofs of Theorems 6.2 and 8.4, and produce iteratively functions $f_{n} \in h^{\infty}(\mathbb{D})$, radii $r_{n}$, positive constants $C_{n}$, and compact subsets $E_{n}^{\sharp, \cup}$, as follows. Along the way, we will define increasing functions $u_{0, n}$ : $\left[0,1\left[\rightarrow\left[0,+\infty\left[\right.\right.\right.\right.$, which tend to the desired function $u_{0}$ as $n \rightarrow+\infty$. We start with $f_{0}=0, r_{0}=\frac{1}{2}, C_{0}=1$, and $E_{0}^{\sharp, \cup}=\emptyset$. We also set $u_{0,0}(t)=0$ on [0,1[. In general, the radius $r_{n}$ will be chosen such that $r_{n-1}<r_{n}<1$, and such that the set $E_{n-1}^{\sharp, \cup}$ is contained in the disk $|z|<r_{n}$. Moreover, as $n \rightarrow+\infty$, we want $r_{n} \rightarrow 1$. Suppose we have $f_{n-1}$ and $E_{n-1}^{\sharp, \cup}$, such that

$$
\begin{gathered}
-v(|z|)-1+2^{-n+1} \leq f_{n-1}(z) \leq u(|z|)-u_{0, n-1}(|z|)+C-2^{-n+1}, \quad z \in \mathbb{D}, \\
u(|z|)-u_{0, n-1}(|z|)-C+2^{-n+1} \leq f(z), \quad z \in E_{n-1}^{\sharp, \cup}, \\
\int_{\mathbb{D}} \exp \left(f_{n-1}(z)\right) d S(z) \leq C_{n-1} .
\end{gathered}
$$

If $N(n)$ is large enough, Lemma 10.1, with $\varepsilon=2^{-n}$ and $\xi=\xi_{n}=2 \log n$, will then deliver a radius $R=R_{n}$ with $r_{n}<R_{n}<1$, a compact set

$$
E_{n}^{\sharp}=\left\{r e^{i \theta}: r_{N(n)}^{\sharp} \leq r \leq r_{N(n)}^{*}, \quad e^{i \theta} \in \bigcup_{j} \bar{I}_{j}\left(\frac{1}{2} \alpha, N(n)\right)\right\},
$$

contained in the ring $R_{n}<|z|<1$, and a function $g=f_{n} \in h^{\infty}(\mathbb{D})$ such that (use a slightly different constant than in the lemma)

$$
\begin{gathered}
-v(|z|)-1+2^{-n} \leq f_{n}(z) \leq u(|z|)-u_{0, n-1}(|z|)+C-2^{-n}, \quad|z|<R_{n}, \\
-v(|z|) \leq f_{n}(z) \leq u(|z|)-\xi_{n}-1+C, \quad R_{n} \leq|z|<1, \\
u(|z|)-u_{0, n-1}(|z|)-C+2^{-n} \leq f_{n}(z), \quad z \in E_{n-1}^{\sharp, \cup}, \\
u(|z|)-\xi_{n}-C+1 \leq f_{n}(z), \quad z \in E_{n}^{\sharp}, \\
\int_{\mathbb{D}} \exp \left(f_{n}(z)\right) d S(z) \leq C_{n},
\end{gathered}
$$

where $C_{n}=C e^{-\xi_{n}}+\exp \left(2^{-n}\right) C_{n-1}$. Declare $u_{0, n}(t)$ to be $u_{0, n-1}(t)$ for $0 \leq t<R_{n}$, and $\xi_{n}=2 \log n$ for $R_{n} \leq t<1$. It is readily checked that this defines an increasing 
function. Also, put $E_{n}^{\sharp, \cup}=E_{n-1}^{\sharp, \cup} \cup E_{n}^{\sharp}$. Part of the above estimates then simplify,

$$
\begin{gathered}
-v(|z|)-1+2^{-n} \leq f_{n}(z) \leq u(|z|)-u_{0, n}(|z|)+C-2^{-n}, \quad z \in \mathbb{D}, \\
u(|z|)-u_{0, n-1}(|z|)-C+2^{-n} \leq f_{n}(z), \quad z \in E_{n}^{\sharp, \cup} .
\end{gathered}
$$

As $n \rightarrow+\infty$, the functions $f_{n} \in h^{\infty}(\mathbb{D})$ converge, uniformly on compact subsets of $\mathbb{D}$, to a function $f$, harmonic in $\mathbb{D}$, and the functions $u_{0, n}$ converge to a function $u_{0}$. Since $\sum_{n} e^{-\xi_{n}}=\sum_{n} n^{-2}$ converges, also the constants $C_{n}$ converge to a limit $C_{\infty}$, so that in the limit, one has

$$
\begin{aligned}
-v(|z|)-1 \leq & f(z) \leq u(|z|)-u_{0}(|z|)+C, \quad z \in \mathbb{D}, \\
u(|z|)- & u_{0}(|z|)-C \leq f(z), \quad z \in E^{\sharp, \cup}, \\
& \int_{\mathbb{D}} \exp (f(z)) d S(z) \leq C_{\infty},
\end{aligned}
$$

with $E^{\sharp, \cup}=\bigcup_{j=1}^{\infty} E_{j}^{\sharp}$. For rapidly increasing $N(n)$, the $R(n)$, being contained between $r_{N(n-1)}^{*}$ and $r_{N(n)}^{*}$, tend to 1 very rapidly in $n$, so that we can make $u_{0}(t)$ go to $+\infty$ as slowly as we like as $t \rightarrow 1$. In particular, we can get $u_{0}(t)=o(u(t))$ as $t \rightarrow 1$.

We now turn to the assertion that

$$
\limsup _{r \rightarrow 1}\left(\frac{1}{r} \int_{r \mathbb{T}} f^{-}(z)-\lambda u_{0}(r)\right)=+\infty .
$$

Since $f$ is big and positive on $E^{\sharp, \cup}$, the integrals

$$
\frac{1}{r} \int_{r \mathbb{T}} f^{+}(z) d s(z)
$$

are correspondingly big for $r=r_{N(n)}^{*}$, and the order of magnitude is at least a positive constant times $u\left(r_{N(n)}^{*}\right)$. By the mean value theorem for harmonic functions, the integrals where $f^{+}$is replaced by $f^{-}$are of the same magnitude, whence the assertion follows.

Finally, we look at the part of the assertion involving the function $h$. Since $f$ has the bound from below, the integrability of $\exp (f+h)$ on the unit disk forces the subharmonic function to satisfy (10-10), for some $\gamma=\gamma(\alpha, \beta)$, by the meanvalue property of subharmonic functions on disks. In fact, if we take the liberty to subtract off a suitable absolute constant from $h$, we can get $\gamma=\beta+2$. Moreover, by the way the function $u_{0}$ was defined in terms of $\xi_{n}$, and the control from below on $f$ on $E^{\sharp, \cup},(10-11)$ holds for some $A=A(\alpha)$. So, we can apply Lemma 10.2, and get the desired estimate, by replacing the $\xi_{n}$ with the appropriate expression in terms of $u_{0}$. The proof is complete.

Remark 10.4. (a) A more careful analysis of the construction part of the demonstration of Theorem 10.3 shows that the harmonic function $f$ constructed has the additional property that the associated function $M[f](r)=\sup _{|z|=r} f(z)$ is exponentially summable, that is, $\exp (M[f])$ is in $L^{1}(0,1)$. It then follows, for instance, that $\exp (f) d S$ is a Carleson measure on $\mathbb{D}$.

(b) For the part of the theorem involving the subharmonic function $h$, by replacing the use of the exponential function in Jensen's inequality with the function $\vartheta_{\gamma}(t)=t^{\gamma}$ for $t>0$, and $\vartheta_{\gamma}(t)=0$ for $t \leq 0$, with exponent $\gamma$ bigger than 1 (so 
that it is convex), we see that for $0<\sigma<1$, the conclusion can be sharpened to say that the integrals

$$
\frac{1}{r} \int_{r \mathbb{T}} \exp (\sigma h(z)) d s(z)
$$

(with $0<r<1$ ) are bounded by expressions of the type

$$
C \exp \left(C u_{0}(r)\right),
$$

where the constant $C=C(\alpha, \sigma)$ is positive.

\section{Construction of extremally Growing functions, IV}

We draw a conclusion from Theorem 10.3 which will be used to prove Theorem 1.4 .

Corollary 11.1. Let $\beta>0$ be real. Then there are an increasing function $u_{0}$ : $\left[0,1\left[\rightarrow\left[0,+\infty\left[\right.\right.\right.\right.$, with $u_{0}(t)=o(-\log (1-t))$ as $t \rightarrow 1$ and $\lim _{t \rightarrow 1} u_{0}(t)=+\infty$, and a function $F$ holomorphic on $\mathbb{D}$, such that

1. $\int_{\mathbb{D}}|F(z)| d S(z)<+\infty$,

2. $(1-|z|)^{\beta} \leq|F(z)|$ for all $z \in \mathbb{D}$,

3. $\lim \sup _{r \rightarrow 1}\left((2 \pi)^{-1} \int_{-\pi}^{\pi} \log ^{-}\left|F\left(r e^{i \theta}\right)\right| d \theta-\lambda u_{0}(r)\right)=+\infty$ for all $\lambda>0$, and

4. if $h$ is subharmonic and bounded from above on $\mathbb{D}$, and has

$\int_{\mathbb{D}}|F(z)| \exp (h(z)) d S(z)=A<+\infty$, then

$(2 \pi)^{-1} \int_{-\pi}^{\pi} h^{+}\left(r e^{i \theta}\right) d \theta \leq \log ^{+} A+C u_{0}(r)$, for $0<r<1$, with a constant $C$ that does not depend on $h$ and $A$.

Moreover, an $F$ can be found with the additional property that

5. $|F(z)| \leq C(1-|z|)^{-1} \exp \left(-u_{0}(|z|)\right)$ on $\mathbb{D}$, for some positive constant $C$.

\section{Proofs of the main theorems}

Let $\mathcal{O}(\mathbb{D})$ denote the Fréchet space of all holomorphic functions on $\mathbb{D}$, with the usual topology of uniform convergence on compact subsets.

Proof of Theorem 1.2. Let $F$ be an outer function in the Nevanlinna class appearing in Corollary 5.1, with $u(r)=p(1-\log (1-r))$ and $v(r)=1+\log \log \left(2+(1-r)^{-1}\right)$. Then $F \in A^{-p}$, and $1 / F \in \bigcap_{q} A^{-q}$. Let $p_{k}$ be a sequence of polynomials, and suppose that $F p_{k}$ tends to 1 in the weak-star topology of $A^{-p}$. But then $F p_{k}$ is uniformly norm-bounded in $A^{-p}$, so that since $|F|$ is maximally large on the sequence $Z$, each $p_{k}$ is uniformly bounded on $Z$, by a constant that does not depend on $k$. It follows from the maximum principle that the polynomials $p_{k}$ are uniformly bounded in $H^{\infty}$. This, however, contradicts the assumption that $F p_{k}$ tends to 1 , because $F$ fails to be bounded away from 0 . This shows that the function $f=F$ works.

Proof of Theorem 1.3. Let $F$ be the function appearing in Corollary 7.1, with $\beta=$ $q / p$. Then the function $f=F^{p}$ is in $A^{-p}$, and $|f(z)| \geq(1-|z|)^{q}$. We intend to show that the weak-star closed invariant subspace of $A^{-p}$ generated by $f$ equals $f H^{\infty}$. Let $g_{k}$ be a sequence of functions in $H^{\infty}$, such that $f g_{k}$ converges in the weak-star topology of $A^{-p}$, which requires that the functions are uniformly norm-bounded in $A^{-p}$, and that they converge in the topology of $\mathcal{O}(\mathbb{D})$. Since the function $f$ is maximally big on the set $E=E(F)$, each function $g_{k}$ is uniformly bounded on $E$, 
by a constant that is independent of $k$. The fact that $E$ is dominating for $H^{\infty}$ entails that the functions $g_{k}$ are uniformly bounded in $H^{\infty}$. It follows that $f H^{\infty}$ is weak-star sequentially closed, and hence weak-star closed. It is therefore the weak-star closed invariant subspace generated by $f$. That $f H^{\infty}$ is not all of $A^{-p}$ follows, for instance, from Remark 7.2.

Proof of Theorem 1.1. Let $F$ be function in Corollary 9.1, with $\beta=q / p$. Then the function $f=F^{p}$ is in $A_{0}^{-p}$, and $1 / f$ is in $A^{-q}$. We shall show that $f$ is not cyclic in $A_{0}^{-p}$. Let $g_{k}$ be a sequence of functions in $H^{\infty}$, such that $f g_{k}$ converges in norm in $A_{0}^{-p}$. Since $f$ grows almost maximally on $E$, we have that $\left|g_{k}(z)\right| \leq$ $C \exp \left(u_{0}(|z|)\right)$ on $E$ for some positive constant $C$. But then, by property 5 of Corollary 9.1, applied to the subharmonic functions $\log \left|g_{k}\right|-\log C$, it follows that $\left|g_{k}(z)\right| \leq C^{\prime} \exp \left(u_{0}(|z|)\right)$ throughout $\mathbb{D}$, for another constant $C^{\prime}$. By property 4 of Corollary $9.1, f g_{k}$ cannot tend to 1 , whence $f$ fails to be cyclic.

Proof of Theorem 1.4. Let $F$ be as in Corollary 11.1, with $\beta=p q$. Then the function $f=F^{1 / p}$ is in $L_{a}^{p}$, and $1 / f$ is in $A^{-q}$. We intend to demonstrate that $f$ is non-cyclic in $L_{a}^{p}$. Let $g_{k}$ be a sequence of functions in $H^{\infty}$ such that $f g_{k}$ converges in norm in $L_{a}^{p}$. By property 4 of Corollary 11.1 applied to the functions $\log \left|g_{k}\right|$, we have that

$$
\frac{1}{2 \pi} \int_{-\pi}^{\pi} \log ^{+}\left|g_{k}\left(r e^{i \theta}\right)\right| d \theta \leq C\left(1+u_{0}(r)\right), \quad 0<r<1,
$$

for a positive constant $C$. By property 3 of Corollary 11.1, it follows that if $g$ is the limit in $\mathcal{O}(\mathbb{D})$ of the sequence $g_{k}$, then

$$
\limsup _{r \rightarrow 1} \frac{1}{2 \pi} \int_{-\pi}^{\pi}\left(\log ^{-}\left|f\left(r e^{i \theta}\right)\right|-\log ^{+}\left|g\left(r e^{i \theta}\right)\right|\right) d \theta=+\infty .
$$

This, however, cannot be if the limit $f g$ is the constant function 1 , for then $\log ^{-}|f|=\log ^{+}|g|$.

\section{Applichtions to inner divisors in the Bergman spaces}

In [16], Boris Korenblum introduced the notion of an outer function in $L_{a}^{p}$ in terms of domination, and proved that a cyclic function necessarily is outer. Recent work due to Alexandru Aleman, Stefan Richter, and Carl Sundberg [2] shows that the outer functions are precisely the cyclic functions. Korenblum [16] anticipated that this would be so, and asked furthermore whether the classes of outer functions and of cyclic functions in $L_{a}^{p}$ might both coincide with the class of functions in $L_{a}^{p}$ that are cyclic in $A^{-\infty}$. By Theorem 1.4, this is not the case.

Another application of Theorem 1.4 concerns the factorization theory for the Bergman spaces in a more direct way. Fix the parameter $p, 0<p<+\infty$, and let $\mathcal{M}$ be a proper closed subspace of $L_{a}^{p}$, which is invariant under multiplication by $z$. Let $m$ be the order of the common zero at the origin of the functions in $\mathcal{M}$, and consider the extremal problem

$$
\sup \left\{\operatorname{Re} f^{(m)}(0): f \in \mathcal{M},\|f\|_{L_{a}^{p}}=1\right\} .
$$

Suppose that $\mathcal{M}$ is singly generated, and that the generator is $f \in L_{a}^{p}$. Some notation: given a function $g \in L_{a}^{p}$, we write $[g]$ for the invariant subspace generated by $g$, and observe that it is obtained as the closure of the set of polynomial multiples of $g$; the assumption on $\mathcal{M}$ therefore reads $\mathcal{M}=[f]$. The above extremal problem 
then has a unique solution, which is denoted by $G=G_{\mathcal{M}}$, and referred to as the extremal function (or canonical divisor) for $\mathcal{M}$. It is shown in [2] (see also [7], [8], [11], [12]) that $\mathcal{M}=[G]$, and that $G$ is a contractive divisor on $\mathcal{M}$ :

$$
\|g / G\|_{L_{a}^{p}} \leq\|g\|_{L_{a}^{p}}, \quad g \in \mathcal{M} .
$$

The following question pops up: Is $G$ an expansive multiplier on all of $L_{a}^{p}$,

$$
\|g\|_{L_{a}^{p}} \leq\|G g\|_{L_{a}^{p}}, \quad g \in L_{a}^{p} ?
$$

A closely related question is the following one: Is it true that

$$
G L_{a}^{p} \cap L_{a}^{p}=[G]=\mathcal{M} ?
$$

To answer both questions in the negative, we use as $f$ the function of Theorem 1.4, for some positive value of the parameter $q$, say $q=1$.

Since $f$ is non-cyclic, $\mathcal{M}=[f]$ is not all of $L_{a}^{p}$. By (13-2), $G$ has no zeros in $\mathbb{D}$, and $f / G \in L_{a}^{p}$. Because $1 / f \in A^{-q}, G$ cannot decrease very quickly radially, and quantitatively, we have, for some positive constants $\sigma$ and $C$,

$$
|G(z)| \geq C(1-|z|)^{\sigma}, \quad z \in \mathbb{D},
$$

that is, $1 / G \in A^{-\infty}$. It follows that the function $\varphi=G^{-\varepsilon}$ is in $L_{a}^{p}$ for some small $\varepsilon, 0<\varepsilon<1$. The function $\varphi G=G^{1-\varepsilon}$ is in $L_{a}^{q}$ for some $q, p<q$, whence $\varphi G$ is cyclic in $L_{a}^{p}$ by Theorem C. The function $\varphi G$ therefore cannot be in $\mathcal{M}$, although $\varphi G \in G L_{a}^{p} \cap L_{a}^{p}$. This answers the second question in the negative.

We turn to the first question. For the same canonical divisor $G=G_{\mathcal{M}}$ and $\varepsilon$ as above, consider the function

$$
L(t)=\int_{\mathbb{D}}\left(|G(z)|^{p}-1\right)|G(z)|^{-t} d S(z), \quad 0 \leq t \leq p \varepsilon,
$$

which is continuous on the indicated interval. Since $G$ has norm 1 , we have $L(0)=0$. The integrand

$$
\left(|G(z)|^{p}-1\right)|G(z)|^{-t}
$$

decreases strictly in the $t$ variable on $0<t<+\infty$ for those values of $z$ where $|G(z)|$ differs from 1 , and equals 0 if $|G(z)|=1$. Since $|G(z)|$ is not a constant function, $L(t)$ must therefore be strictly decreasing on the interval $[0, p \varepsilon[$. In particular, $L(t)<0$ for $0<t \leq p \varepsilon$. But then $\varphi=G^{-\varepsilon}$ has bigger norm than $\varphi G=G^{1-\varepsilon}$ in $L_{a}^{p}$, although $\varphi \in L_{a}^{p}$.

It is possible that given any positive $\sigma$, there are non-constant inner divisors $G$ such that (13-5) holds for some small but positive $C$. This, however, does not follow from the above arguments.

\section{ACKNOWLEDGEMENTS}

Thanks are due to Sergei Treil for assistance with the computer generation of Figure 10-1, and to Donald Marshall and John McCarthy for making helpful suggestions. 


\section{REFERENCES}

[1] A. Aharonov, H. S. Shapiro, A. L. Shields, Weakly invertible elements in the space of square-summable holomorphic functions, J. London Math. Soc. (2) 9 (1974/5), 183-192. MR 51:1403

[2] A. Aleman, S. Richter, C. Sundberg, Beurling's theorem for the Bergman space, preprint 1995.

[3] A. A. Borichev, P. J. H. Hedenmalm, Cyclicity in Bergman-type spaces, Internat. Math. Res. Notices (no. 5) (1995), 253-262. MR 96h:46030

[4] L. Brown, B. I. Korenblum, Cyclic vectors in $A^{-\infty}$, Proc. Amer. Math. Soc. 102 (1988), 137-138. MR 89e:46025

[5] L. Brown, A. L. Shields, K. Zeller, On absolutely convergent exponential sums, Trans. Amer. Math. Soc. 96 (1960), 162-183. MR 26:332

[6] N. Dunford, J. Schwartz, Linear operators, Part I: General Theory, 1958. MR 22:8302

[7] P. Duren, D. Khavinson, H. S. Shapiro, C. Sundberg, Contractive zero-divisors in Bergman spaces, Pacific J. of Math. 157 (1993), 37-56. MR 94c:30048

[8] P. Duren, D. Khavinson, H. S. Shapiro, C. Sundberg, Invariant subspaces in Bergman spaces and the biharmonic equation, Michigan Math. J. 41 (1994), 247-259. MR 95e:46030

[9] R. Frankfurt, Weak invertibility and factorization in certain spaces of analytic functions, Problem 11.12 in Linear and Complex Analysis Problem Book 3, Part II, Lecture Notes in Math. 1574 (1994), 33-35. MR 96c:00001b

[10] J. B. Garnett, Bounded analytic functions, Academic Press, 1981. MR 83g:30037

[11] H. Hedenmalm, A factorization theorem for square area-integrable analytic functions, J. Reine Angew. Math. 422 (1991), 45-68. MR 93c:30053

[12] H. Hedenmalm, Open problems in the function theory of the Bergman space, Festschrift in honour of Lennart Carleson and Yngve Domar (Uppsala, 1993), Acta Univ. Upsaliensis C58, Uppsala, Sweden (1995), pp. 153-169. MR 96j:46023

[13] B. Korenblum, An extension of the Nevanlinna theory, Acta Math. 135 (1975), 187-219. MR 54:13081

[14] B. Korenblum, A Beurling-type theorem, Acta Math. 138 (1977), 265-293. MR 56:5894

[15] B. Korenblum, Weakly invertible elements in Bergman spaces, Problem 11.13 in Linear and Complex Analysis Problem Book 3, Part II, Lecture Notes in Math. 1574 (1994), 36-37. MR 96c:00001b

[16] B. Korenblum, Outer functions and cyclic elements in Bergman spaces, J. Funct. Anal. 115 (1993), 104-118. MR 94h:46038

[17] N. K. Nikolskiř, Selected problems of weighted approximation and spectral analysis, Trudy MIAN 120 (1974); English transl. in Proc. of the Steklov Institute of Math. 120 (1974). MR $\mathbf{5 7 : 7 1 3 3 b}$

[18] K. Seip, Beurling type density theorems in the unit disk, Invent. Math. 113 (1993), 21-39. MR 94g:30033

[19] F. A. Shamoyan, Weak invertibility in some spaces of analytic functions, Dokl. Akad. Nauk Arm. SSR 74 (no. 4) (1982), 157-161. MR 84e:30077

[20] H. S. Shapiro, Weighted polynomial approximation and boundary behavior of analytic functions, Contemporary Problems in Analytic Functions (Proc. Internat. Conference, Erevan, Armenia, 1965), Nauka, Moscow (1966), pp. 326-335. MR 35:383

[21] H. S. Shapiro, Some remarks on weighted polynomial approximation of holomorphic functions, Mat. Sbornik 73 (1967), 320-330; English transl. in Math. USSR Sbornik 2 (1967), 285-294. MR 36:395

[22] A. L. Shields, Weighted shift operators and analytic function theory, Math. Surveys 13: Topics in operator theory, ed. C. M. Pearcy, Amer. Math. Soc., Providence, RI (1974), pp. 49-128. MR 50:14341

[23] A. L. Shields, Cyclic vectors in spaces of analytic functions, Problem 11.14 in Linear and Complex Analysis Problem Book 3, Part II, Lecture Notes in Math. 1574 (1994), 38-40. MR 96c:00001b

[24] A. L. Shields, Cyclic vectors in Banach spaces of analytic functions, Operators and Function Theory, ed. S. C. Power, Reidel Publishing Company (1985), pp. 315-349. MR 87c:47048 
ABSTRACT. In the theory of commutative Banach algebras with unit, an element generates a dense ideal if and only if it is invertible, in which case its Gelfand transform has no zeros, and the ideal it generates is the whole algebra. With varying degrees of success, efforts have been made to extend the validity of this result beyond the context of Banach algebras. For instance, for the Hardy space $H^{2}$ on the unit disk, it is known that all invertible elements are cyclic (an element is cyclic if its polynomial multiples are dense), but cyclic elements need not be invertible. In this paper, we supply examples of functions in the Bergman and uniform Bergman spaces on the unit disk which are invertible, but not cyclic. This answers in the negative questions raised by Shapiro, Nikolskiı̌, Shields, Korenblum, Brown, and Frankfurt.

Department of Mathematics, University of Bordeaux I, 351, cours de la Liberation, 33405 Talence, France

E-mail address: borichev@math.u-bordeaux.fr

Department of Mathematics, Lund University, Box 118, 22100 Lund, Sweden

E-mail address: haakan@maths.1th.se 